\title{
COALESCING BROWNIAN FLOWS: A NEW APPROACH
}

\author{
By Nathanä̈l Berestycki ${ }^{1}$, Christophe GArban ${ }^{2}$ And Arnab Sen \\ University of Cambridge, Université Lyon 1 and University of Minnesota
}

The coalescing Brownian flow on $\mathbb{R}$ is a process which was introduced by Arratia [Coalescing Brownian motions on the line (1979) Univ. Wisconsin, Madison] and Tóth and Werner [Probab. Theory Related Fields 111 (1998) 375-452], and which formally corresponds to starting coalescing Brownian motions from every space-time point. We provide a new state space and topology for this process and obtain an invariance principle for coalescing random walks. This result holds under a finite variance assumption and is thus optimal. In previous works by Fontes et al. [Ann. Probab. 32 (2004) $2857-$ 2883], Newman et al. [Electron. J. Probab. 10 (2005) 21-60], the topology and state-space required a moment of order $3-\varepsilon$ for this convergence to hold. The proof relies crucially on recent work of Schramm and Smirnov on scaling limits of critical percolation in the plane. Our approach is sufficiently simple that we can handle substantially more complicated coalescing flows with little extra work-in particular similar results are obtained in the case of coalescing Brownian motions on the Sierpinski gasket. This is the first such result where the limiting paths do not enjoy the noncrossing property.

\section{Introduction.}

1.1. Motivation. The coalescing Brownian flow or Arratia's flow was first introduced and studied by Arratia [3, 4], as a limiting object describing the largescale behaviour of the one-dimensional voter model. Informally, this process consists of particles that perform independent coalescing Brownian motions, starting from every space time $(x, t)$. By independent coalescing Brownian motions, we mean two paths which are independent Brownian motions until the first time they meet, and which subsequently continue as one single Brownian motion.

Since then, the Brownian web has been conjectured or proved to describe scaling limits in a large number of seemingly disconnected models: let us mention in particular the works of Tóth and Werner [25] in connection with true self-repelling motion, Coletti et al. [10] in connection with a drainage network model, Sarkar

Received November 2013; revised July 2014.

${ }^{1}$ Supported in part by EPSRC Grants EP/GO55068/1 and EP/I03372X/1.

${ }^{2}$ Supported in part by the ANR Grant MAC2 10-BLAN-0123.

MSC2010 subject classifications. 60K35, 82C21, 60F17.

Key words and phrases. Coalescing Brownian motions, coalescing random walks, invariance principle for coalescing random walks, Arratia flow, Brownian web, Schramm-Smirnov space of coalescing flows, coalescing flow on Sierpinski gasket, non-crossing property. 
and Sun [22] in connection with oriented percolation, Norris and Turner [21] in connection with Hastings-Levitov planar aggregation models.

It is not a priori easy to turn the informal description in the first paragraph into a rigorous mathematical object. The difficulty lies in the fact that there are uncountably many starting points. Curiously, foundations for constructing the Brownian web as a random variable in a "nice" space and studying convergence of discrete objects to the Brownian web were only laid down recently, in a series of papers by Fontes et al. [12].

\subsection{Main results. Our object in this paper is threefold:}

1. First, we provide an alternative state-space and topology for coalescing flows such as Arratia's flow (Theorem 3.1). Inspired by the setup introduced by Schramm and Smirnov in [23] for critical percolation, we call this space the SchrammSmirnov space of coalescing flows $\mathscr{H}$. See Definition 2.5. As the reader will see, our setup has the great advantage that it makes the proof of convergence of discrete objects to the limiting Brownian flow surprisingly simple.

2. We then prove an invariance principle (Theorem 4.1) for the convergence of scaled coalescing random walks on $\mathbb{Z}$ toward Arratia's flow under an optimal finite variance assumption on the random walk. Note that in previous works, the topological setup was different and establishing tightness already required a nontrivial proof. In particular, in [7], it is shown that in order to obtain a tightness criterion, a $3+\varepsilon$ finite moment is sufficient, while existence of a $3-\varepsilon$ moment is needed. We prove in our present setting that a finite variance is both necessary and sufficient.

3. Finally, we illustrate the simplicity and flexibility of our approach by showing similar results for coalescing flows where the underlying geometry is substantially more complicated. We focus in particular on coalescing random walks on the discrete infinite Sierpinski gasket, and prove that this can be rescaled to a coalescing Brownian flow on the continuous Sierpinski gasket (Theorem 6.1). On the real line any two continuous paths cannot cross without hitting each other. This is an obvious topological fact in dimension one which underlies Arratia's original approach and much of the work on the subject. But this property is absent for the Sierpinski Gasket since one of the paths can go into some other "triangle" and come back later at a suitable time.

At the heart of our approach is the groundbreaking work of Schramm and Smirnov [23] on scaling limits of critical percolation in the plane. We borrow directly from their work by adapting the quad-crossing or Schramm-Smirnov statespace and topology to the study of coalescing flows. As in the percolation case, the state space is compact, so only uniqueness of subsequential limits has to be established. Thus, one of the main advantages of our approach is that very few estimates are needed in order to establish this convergence. The bulk of the work is in some sense to construct the limiting object in the Schramm-Smirnov space. Convergence of the discrete object to the continuous one then follows rather simply 
by an argument based on "uniform coming down from infinity" (Proposition 4.4), which is the main technical ingredient.

In the companion paper [8], we will illustrate further the extent and breadth of the analogy between coalescing flows and critical percolation, by showing that the Brownian webs in this paper (including, say, on the Sierpinski gasket) are all examples of black noises.

1.3. Relation to previous work. As mentioned earlier, one of the main results in this paper (Theorem 4.1) is an invariance principle for coalescing random walk under a second moment assumption. This contrasts sharply with the approach developed by Fontes et al. [12]: indeed, using that topology, a series of works culminating with Newman et al. [20] showed that such an invariance principle holds a under a fifth moment assumption. Subsequently, Belhaouari et al. [7] lowered this to a $3+\varepsilon$ moment assumption and, surprisingly, showed that this was in fact also necessary, in the sense that the convergence does not hold if the random walk's $3-\varepsilon$ moments are not finite for some $\varepsilon>0$.

To understand better the difference between the two approaches, it is useful to dwell further on the analogy with percolation. In that context, it is also a nontrivial task to build a "nice" state-space and topology for taking scaling limits (as the mesh size tends to 0 ). One can study the collection of all individual contour interfaces of clusters (an approach originating in the work of Aizenmann and Buchard [1] and culminating in the work of Camia and Newman [9]), or one can ask about macroscopic connectivity properties. This is the viewpoint taken in the work of Schramm and Smirnov [23] which motivates our approach. This latter topological framework introduced by Schramm and Smirnov was recently used and extended in [13] and [14] in order to prove that near-critical percolation on the triangular grid has a (massive) scaling limit.

Some alternative approaches to the construction of Arratia's flow have been proposed which we briefly review. One of the problem for formulating a state-space for Arratia's flow is that it is not in the strict sense a flow, for example, it may well be the case that from a point $x \in \mathbb{R}$ there is more than one trajectory coming out of it. To go around this problem, Norris and Turner [21] considered the space of weak flows, which replaces the notion of a flow $\left(\phi_{s t}\right)_{s<t}$ by a pair $\left(\phi_{s t}^{ \pm}\right)_{s<t}$ satisfying some compatibility relations. Essentially, $\phi^{-}$is the left-continuous version of the flow and $\phi^{+}$the right-continuous version. Arratia's flow is then identified as the unique random variable on this space such that its restriction to finitely many points forms a coalescing Brownian motion started from that set. This approach is very elegant but has the drawback that it relies crucially on the noncrossing property.

Le Jan and Raimond, in a series of papers, [18, 19], adopted a different point of view on the question. They viewed Arratia's flow as a (random) flow of maps, which are essentially consistent systems of $n$-point Markovian systems, describing the law of the motion of $n$ indivisible points. While this approach is in principle very general (and in particular, does not rely on the noncrossing property), it is not 
well-suited to the questions of taking a scaling limit of some discrete flow to its continuous counterpart. This is because the question of scaling limits would have to be approached through the finite-dimensional distributions of the flow. See, for instance, [17] for an example and see [16] for a good survey of this approach.

\subsection{Organisation of the paper. The paper will be divided as follows:}

- In Section 2, we describe the setup used in this paper, that is, the SchrammSmirnov space $\mathscr{H}$ (see Definition 2.5) and its topology. We also explain how to view a compact set of paths as an element of the state space $\mathscr{H}$, and give a convenient criterion for convergence in terms of tube-crossing probabilities.

- In Section 3, we give a construction and characterisation of the Brownian web as a random variable on $\mathscr{H}$ (Theorem 3.1). This is immediately followed in Section 3.2 by a result characterising further the Brownian web. This is not needed for the rest of the paper and may be skipped by a reader who only wishes to read the invariance principle of Section 4.

- In Section 4, we state and prove the invariance principle showing that rescaled coalescing random walks converge to the Brownian web (Theorem 4.1).

- In Section 5, we extend our setup to study simple coalescing random walks on the Sierpinski gasket.

- In the final Section 6, we state and prove an invariance principle showing that rescaled coalescing random walks on the Sierpinski graph converge to the coalescing flow on the Sierpinski gasket constructed in Section 5.

- In the Appendix, we make the link with the state-space and topology of Fontes et al. In particular, we show that our topology is coarser.

\section{Schramm-Smirnov topology and flows.}

2.1. The space of tubes. We start by introducing the notion of tubes (which replaces the notion of quads in the case of planar percolation). Fix $d \geq 1$; we will consider in this section coalescing flows for which the individual paths are naturally embedded into $\mathbb{R}^{d}$. We will denote a generic space-time point $z=(x, t)$, where $x \in \mathbb{R}^{d}$ represents the spatial coordinate and $t$ is the time-coordinate.

Definition 2.1 (Tube). A tube $T$ is a triplet $\left([T], \partial_{0} T, \partial_{1} T\right):=(\varphi([0$, $\left.\left.1]^{d+1}\right), \varphi\left([0,1]^{d} \times\{0\}\right), \varphi\left([0,1]^{d} \times\{1\}\right)\right)$ where $\varphi:[0,1]^{d} \times[0,1] \rightarrow \varphi\left([0,1]^{d} \times\right.$ $[0,1]) \subset \mathbb{R}^{d} \times \mathbb{R}$ is a homeomorphism such that $\varphi\left([0,1]^{d} \times\{0\}\right)$ and $\varphi\left([0,1]^{d} \times\right.$ $\{1\})$ are subsets of $\mathbb{R}^{d} \times\left\{t_{0}\right\}$ and $\mathbb{R}^{d} \times\left\{t_{1}\right\}$, respectively, for some $t_{0}<t_{1}$. Furthermore, we require that $[T]=\varphi\left([0,1]^{d+1}\right)$ is included in $\mathbb{R}^{d} \times\left[t_{0}, t_{1}\right]$. Informally, $T$ is a topological cube along with a distinct pair of opposite faces which are both orthogonal in $\mathbb{R}^{d+1}$ to the time axis. We call $t_{0}$ the start time of $T$ and $t_{1}$ the end time of $T$. The sets $\partial_{0} T$ and $\partial_{1} T$ are called the lower face and the upper face of $T$, respectively. See Figure 1 in the case $d=1$. 

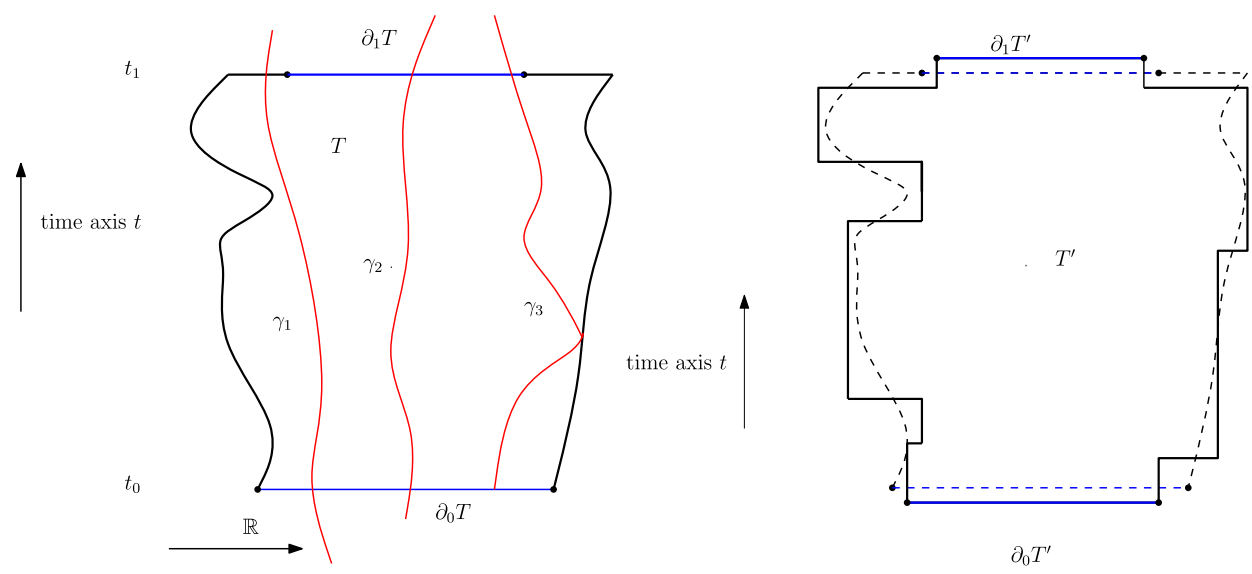

FIG. 1. On the left, a tube $T$ with lower and upper face $\partial_{0} T$ and $\partial_{1} T$. The tube $T$ is traversed by paths $\gamma_{2}$ and $\gamma_{3}$ but not by $\gamma_{1}$. On the right, a tube $T^{\prime}$ from the class $\mathcal{T}_{\square}$ of Definition 2.14 approximating a tube $T$, shown in dotted line.

DEFinition 2.2 (Metric space of tubes). The space of all such tubes, denoted by $\mathcal{T}$, can be equipped with the following metric:

$$
d_{\mathcal{T}}\left(T_{1}, T_{2}\right):=d_{\text {Haus }}\left(\left[T_{1}\right],\left[T_{2}\right]\right)+d_{\text {Haus }}\left(\partial_{0} T_{1}, \partial_{0} T_{2}\right)+d_{\text {Haus }}\left(\partial_{1} T_{1}, \partial_{1} T_{2}\right),
$$

where $d_{\text {Haus }}$ is the usual Hausdorff metric on the compact subsets of $\mathbb{R}^{d+1}$, which is given by $d_{\text {Haus }}(A, B)=\max \left(\sup _{b \in B} \inf _{a \in A}\|a-b\|_{2}, \sup _{a \in A} \inf _{b \in B}\|a-b\|_{2}\right)$. It is easy to see that $\left(\mathcal{T}, d_{\mathcal{T}}\right.$ ) is separable (see, e.g., Section 2.6.1).

2.2. The space of coalescing flows. First, we introduce the notion of crossing or traversing for a tube by a continuous path in $\mathbb{R}^{d}$ with a specified starting time. A continuous path $\gamma$ with starting time $t$ is just a continuous map $\gamma:[t, \infty) \rightarrow \mathbb{R}^{d}$ and is denoted by $(\gamma, t)$. We will say that a tube $T$ is crossed or traversed by $(\gamma, t)$ if $t \leq t_{0},\left(\gamma\left(t_{0}\right), t_{0}\right) \in \partial_{0} T,\left(\gamma\left(t_{1}\right), t_{1}\right) \in \partial_{1} T$ and $(\gamma(s), s) \in[T]$ for all $s \in\left(t_{0}, t_{1}\right)$, where $t_{0}$ and $t_{1}$ are the start time and the end time of $T$, respectively. Informally, this means that the trajectory of the path $\gamma$ enters the tube $T$ through the lower face $\partial_{0} T$ and stays in it throughout until it exits $T$ through its upper face $\partial_{1} T$. Given any (finite or infinite) collection of such continuous paths with starting times, we can associate the subset of $\mathcal{T}$ which consists of all the tubes which are crossed by at least one path from the collection. For example, in the case $d=1$, for a family of continuous (random) paths which are trajectories of some coalescing Brownian particles starting from different space-time points in $\mathbb{R}^{2}$, we can naturally obtain via the above association an element of $\{0,1\}^{\mathcal{T}}$. This contains a lot of information about the coalescing paths. This suggests taking $\{0,1\}^{\mathcal{T}}$ as a state-space for the Brownian web. But as indicated in [23], this turns out to be too big and unwieldy for our purpose. Instead, note that a subset of $\mathcal{T}$ consisting of all the tubes which 


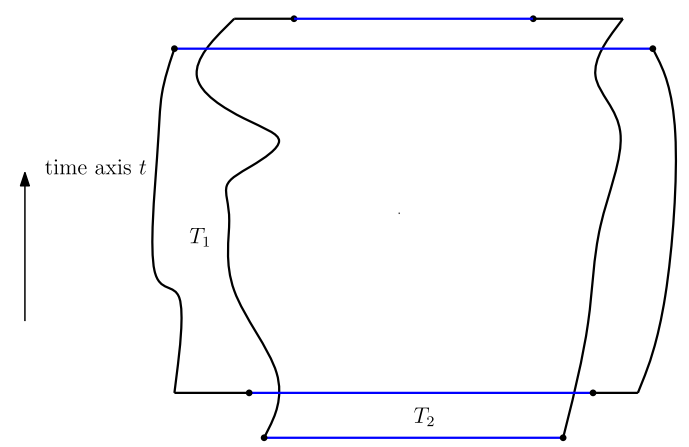

FIG. 2. Two tubes such that $T_{1}<T_{2}$.

are crossed by some collection of paths cannot be arbitrary: some compatibility conditions must be satisfied. The following partial order on tubes, which mirrors that of [23] will help to clarify the structure.

DEFinition 2.3 (Partial orders on tubes). If $T_{1}, T_{2} \in \mathcal{T}$ are two tubes, we will say that:

(a) $T_{1} \leq T_{2}$ if whenever $T_{2}$ is traversed by any path $\gamma, T_{1}$ is also traversed by $\gamma$. See Figure 2.

(b) $T_{1}<T_{2}$ if there are open neighborhoods $U_{i}$ of $T_{i}$ in $\left(\mathcal{T}, d_{\mathcal{T}}\right)$ such that $T_{1}^{\prime} \leq$ $T_{2}^{\prime}$ holds for any $T_{i}^{\prime} \in U_{i}, i=1,2$.

The definition below (following again [23]) singles out a particular class of subsets of $\mathcal{T}$ by adding the compatibility constraint on the tubes which are considered to be traversed.

DEFINITION 2.4 (Hereditary subsets of $\mathcal{T}$ ). A subset $S \subset \mathcal{T}$ is called hereditary if whenever $T \in S$ and $T^{\prime} \in \mathcal{T}$ such that $T^{\prime}<T$, we also have $T^{\prime} \in S$.

We are now ready to define the crucial definition for our state-space:

DEFinition 2.5 (Space of coalescing flows). Let $\mathscr{H}$ be the space of all closed hereditary subsets of $\mathcal{T}$. We call $\mathscr{H}$ the Schramm-Smirnov space of coalescing flows or simply the space of coalescing flows.

REMARK 2.6. The word closed in the above definition refers to the Hausdorff metric on tubes of Definition 2.2. Essentially, it is a way of setting the following convention: if $T_{n} \rightarrow T$ in $d_{\mathcal{T}}$ and each $T_{n}$ is crossed by a family of continuous paths, then we declare $T$ to be crossed by that family. Another way to think about it is that it shows how to decide whether a tube is crossed in the ambiguous case when a path touches the boundary of the tube, like the path $\gamma_{3}$ in Figure 1. Such a tube is declared crossed. 
2.3. The tube-topology. Having defined our state-space $\mathscr{H}$ we need a convenient topology for it. Following [23], we define a topology on $\mathscr{H}$ generated by the following subbase (their finite intersections form a basis for the topology)

$$
\begin{gathered}
\{H \in \mathscr{H}: H \cap U \neq \varnothing\}, \quad U \subseteq \mathcal{T} \text { topologically open, } \\
\{H \in \mathscr{H}: T \notin H\}, \quad T \in \mathcal{T} .
\end{gathered}
$$

We denote $\{H \in \mathscr{H}: T \in H\}$ and $\{H \in \mathscr{H}: H \cap U \neq \varnothing\}$ by $\boxminus_{T}$ and $\boxminus^{U}$, respectively.

Intuitively speaking, this choice of open sets for the tube-topology leads to the following desirable properties: if a tube $T$ is crossed by a collection of paths, and if those paths undergo a slight 'perturbation', then some tube in the neighborhood of $T$ will still be crossed [corresponding to (2.1)]. Also, if $T$ is not crossed by a collection of paths, then a small perturbation in those paths cannot result in a crossing of $T$ [corresponding to (2.2)].

The following fundamental result is due to Schramm and Smirnov.

THEOREM 2.7. The Schramm-Smirnov space $\mathscr{H}$ equipped with the tubetopology has the following properties:

1. $\mathscr{H}$ is separable, metrizable, and hence a Polish space.

2. $\mathscr{H}$ is compact.

3. For any dense set $\mathcal{S}$ of $\mathcal{T}$, the $\sigma$-field generated by $\left\{\boxminus_{T}: T \in \mathcal{S}\right\}$ is the Borel $\sigma$-field on $\mathscr{H}$.

The above theorem is an immediate consequence of a more general result (precisely Theorem 3.10) of [23] which works for the space of closed hereditary subsets of any second-countable topological space $X$ with a partial ordering $\prec$ such that the ordering satisfies

$$
\left\{(x, y) \in X^{2}: x \prec y\right\} \quad \text { is a topologically open subset of } X^{2},
$$

and

$$
\forall x \in X, \quad x \in \overline{\{y \in X: y \prec x\}} .
$$

For us, $X=\mathcal{T}$ with the partial order $<$ in which case conditions (2.3) and (2.4) are easy to verify.

The reader may find close similarities between the tube-topology and the standard Fell topology (or the topology for closed convergence) defined on $\operatorname{Cld}(\mathcal{T})$, the space of closed subsets of $\mathcal{T}$. The Fell topology has many desirable properties including compactness [which is lacked by the topology induced by the Hausdorff metric on $\mathrm{Cld}(\mathcal{T})$ ]. The fact is using (2.3) and (2.4), one can prove that $\mathscr{H}$ is closed subset of $\operatorname{Cld}(\mathcal{T})$ under the Fell topology, and moreover, the induced topology on $\mathscr{H}$ is the same as the tube-topology.

Later we will need the next lemma which says that a monotone (with respect to set inclusion) sequence in $\mathscr{H}$ always converges. 
Lemma 2.8. Let $H_{1} \subseteq H_{2} \subseteq \cdots$ be a nondecreasing sequence in $\mathscr{H}$. Then there exists $H \in \mathscr{H}$ such that $d_{\mathscr{H}}\left(H_{n}, H\right) \rightarrow 0$. Furthermore, $H=\overline{\bigcup_{n=1}^{\infty} H_{n}}$, the closure being taken with respect to $d_{\mathcal{T}}$.

Proof. Since $\mathscr{H}$ is compact, $H_{k}$ has subsequential limits. Let $H, H^{\prime}$ be two such limits, taken, respectively, along the subsequences $\left(n_{k}\right)$ and $\left(m_{j}\right)$. Let us show first that $H_{n} \subseteq H$ for all $n \geq 1$. If $T$ is a tube such that $T \in H_{n}$ for some $n$ then by monotonicity $T \in H_{n_{k}}$ for all $k$ sufficiently large. In other words, $H_{n_{k}} \in \boxminus_{T}$ for all sufficiently large $k$. Since $\boxminus_{T}$ is a closed set in $\left(\mathscr{H}, d_{\mathscr{H}}\right)$, we have $H=$ $\lim _{k} H_{n_{k}} \in \boxminus_{T}$. Hence, $T \in H$ as well.

Now let us show that $H^{\prime} \subseteq H$. Fix $T \in H^{\prime}$. By definition of the basic open sets given in (2.1), for all $\delta>0$ there exists $T_{\delta} \in \mathcal{T}$ such that $d_{\mathcal{T}}\left(T, T_{\delta}\right)<\delta$ and $T_{\delta} \in H_{m_{j}}$ for all sufficiently large $j$. Since $H_{m_{j}} \subseteq H$ by the above observation, $T_{\delta} \in H$ for all $\delta$. But now since $H$ is a closed subset of $\mathcal{T}$ and $d_{\mathcal{T}}\left(T_{\delta}, T\right) \rightarrow 0$ as $\delta \rightarrow 0$, it follows that $T \in H$ as well. Hence, $H^{\prime} \subseteq H$. By symmetry $H=H^{\prime}$ and this proves the uniqueness of the subsequential limit.

Now since $H_{k} \subseteq H$ for all $k \geq 1$ and $H$ is closed, we have $\overline{\bigcup_{k=1}^{\infty} H_{k}} \subseteq H$. We have also shown that given $T \in H$ there exists a sequence of tubes $T_{\delta} \in \bigcup_{k=1}^{\infty} H_{k}$ such that $d_{\mathcal{T}}\left(T_{\delta}, T\right) \rightarrow 0$ as $\delta \rightarrow 0$. This shows that $H \subseteq \overline{\bigcup_{k=1}^{\infty} H_{k}}$ and the proof of the lemma is complete.

2.4. Coalescing paths as an element of the space $\mathscr{H}$. Let $C\left[t_{0}\right]$ denote the set of continuous functions from $\left[t_{0}, \infty\right)$ to $\mathbb{R}^{d}$. Define

$$
\Pi=\bigcup_{t_{0} \in \mathbb{R}} C\left[t_{0}\right] \times\left\{t_{0}\right\},
$$

where $\left(\gamma, t_{0}\right) \in \Pi$ represents a path $\gamma$ in $\mathbb{R}^{d}$ which starts from time $t_{0}$. For $\left(\gamma, t_{0}\right) \in$ $\Pi$, let $\hat{\gamma}$ be the continuous function that extends $\gamma$ to entire time $\mathbb{R}$ by setting $\hat{\gamma}(t)=\gamma\left(t_{0}\right)$ for all $t<t_{0}$. We then define a distance $\varrho$ on $\Pi$ by

$$
\varrho\left(\left(\gamma_{1}, t_{1}\right),\left(\gamma_{2}, \gamma_{2}\right)\right)=d_{\text {sup }}\left(\hat{\gamma}_{1}, \hat{\gamma}_{2}\right)+\left|t_{1}-t_{2}\right|,
$$

where $d_{\text {sup }}\left(\hat{\gamma}_{1}, \hat{\gamma}_{2}\right)=\sum_{k=1}^{\infty} 2^{-k} \sup _{t \in[-k, k]} \min \left(\left\|\hat{\gamma}_{1}(t)-\hat{\gamma}_{2}(t)\right\|_{2}, 1\right)$. It is easy to check that $(\Pi, \varrho)$ is a complete separable metric space.

If $\zeta \subseteq \Pi$ is any collection of continuous functions (with starting times), one can naturally associate to it a subset $\operatorname{Cr}(\zeta)$ of $\mathcal{T}$ consisting of all tubes traversed by $\zeta$. It is clear that $\operatorname{Cr}(\zeta)$ is hereditary for any $\zeta \subseteq \Pi$. To associate to $\zeta$ a genuine member of $\mathscr{H}$ we need to check that $\operatorname{Cr}(\zeta)$ is closed in $\mathcal{T}$ so that $\operatorname{Cr}(\zeta) \in \mathscr{H}$. The following straightforward lemma says that this happens at least if $\zeta$ is compact.

Lemma 2.9. If $\zeta \subseteq \Pi$ is compact, then $\operatorname{Cr}(\zeta) \in \mathscr{H}$. 
Proof. We need to show that if $T_{n} \in \operatorname{Cr}(\zeta)$ and $d_{\mathcal{T}}\left(T_{n}, T\right) \rightarrow 0$, then $T \in$ $\operatorname{Cr}(\zeta)$. Suppose that $T_{n}$ is traversed by $\left(\gamma_{n}, \tau_{n}\right) \in \zeta$. Since $\zeta$ is compact, we can find a subsequence $\left\{n_{k}\right\}$ such that $\left(\gamma_{n_{k}}, \tau_{n_{k}}\right) \rightarrow(\gamma, \tau) \in \zeta$ in the metric $\varrho$. Let $t_{0}\left(T_{n}\right)$ and $t_{0}(T)$ be the starting times of the tubes $T_{n}$ and $T$, respectively. Their ending times $t_{1}\left(T_{n}\right)$ and $t_{1}(T)$ are defined similarly. Note that $t_{i}\left(T_{n_{k}}\right) \rightarrow t_{i}(T)$, for $i=0,1$.

Since $\tau_{n_{k}} \leq t_{0}\left(T_{n_{k}}\right)$, we have $\tau \leq t_{0}(T)$. We first need to show that $(\gamma(s), s) \in$ [T] for each $s \in\left(t_{0}(T), t_{1}(T)\right)$. Fix $s \in\left(t_{0}(T), t_{1}(T)\right)$. Since we can find $s_{n_{k}} \in$ $\left[t_{0}\left(T_{n_{k}}\right), t_{1}\left(T_{n_{k}}\right)\right]$ such that $s_{n_{k}} \rightarrow s$. Then $\left(\gamma_{n_{k}}\left(s_{n_{k}}\right), s_{n_{k}}\right) \in\left[T_{n_{k}}\right]$. By uniform convergence of $\gamma_{n_{k}}$ to $\gamma$ on the compact time intervals, we have $\gamma_{n_{k}}\left(s_{n_{k}}\right) \rightarrow \gamma(s)$, and hence $(\gamma(s), s) \in[T]$. Since $\left(\gamma_{n_{k}}\left(t_{i}\left(T_{n_{k}}\right)\right), t_{i}\left(T_{n_{k}}\right)\right) \rightarrow\left(\gamma\left(t_{i}(T)\right), t_{i}(T)\right)$ for each $i$, we can easily see that $\left(\gamma\left(t_{i}\right), t_{i}\right) \in \partial_{i} T$ for $i=1,2$. This completes the proof.

REMARK 2.10. The conclusion of the above lemma holds under the following slightly weaker assumption which turns out to be convenient in practice. Let $T$ be a tube and for $\left(\gamma, t_{0}\right) \in \Pi$ set $\tau(\gamma, T):=\inf \left\{t \geq t_{0}: \gamma(t) \in \partial_{0} T\right\}$ (with $\inf \varnothing=+\infty$, as usual), and set $\gamma_{T}(t)=\gamma(t)$ for $t \geq \tau(\gamma, T)$. If $\zeta \subseteq \Pi$ set $\zeta_{T}=\left\{\left(\gamma_{T}, \tau(\gamma, T)\right): \tau(\gamma, T)<\infty\right\}$. Then the conclusion of Lemma 2.9 holds as soon as $\zeta_{T}$ is compact in $\Pi$ for every tube $T \in \mathcal{T}$. In words, if the collection of paths $\zeta$, restricted to any particular tube $T$, is compact, then this induces an element $\operatorname{Cr}(\zeta) \in \mathscr{H}$.

2.5. Countable coalescing process. Fix $d \geq 1$ and a countable ordered set of $\mathbb{R}^{d}$-valued continuous paths $\left(\gamma_{j}, s_{j}\right)_{j \geq 1}$ in $\Pi$. From this countable set of free paths, we define an ordered set of coalescing paths $\left(\gamma_{j}^{c}, s_{j}\right)_{j \geq 1}$ in $\Pi$ inductively using the following coalescing rule. Set $\gamma_{1}^{c}=\gamma_{1}$. For $j>1$, set

$$
\tau_{j}=\inf \left\{t \geq s_{j}: \gamma_{j}(t) \in\left\{\gamma_{1}^{c}(t), \gamma_{2}^{c}(t), \ldots, \gamma_{j-1}^{c}(t)\right\}\right\}
$$

with the usual convention that inf $\varnothing=\infty$. Take

$$
I_{j}=\min \left\{i \in\{1,2, \ldots, j-1\}: \gamma_{j}\left(\tau_{j}\right)=\gamma_{i}^{c}\left(\tau_{j}\right)\right\} \quad \text { if } \tau_{j}<\infty .
$$

For $t \geq s_{j}$, define

$$
\gamma_{j}^{c}(t)= \begin{cases}\gamma_{j}(t), & \text { if } t<\tau_{j}, \\ \gamma_{I_{j}}^{c}(t), & \text { if } t \geq \tau_{j} .\end{cases}
$$

In words, if the free paths of labels $i$ and $j$ collide, they both subsequently follow the path with the lower label.

2.6. Characterization and convergence criterion for probability measures on $\mathscr{H}$. A general tube can be extremely complicated and its crossing probability can be very hard to deal with. But in order to characterize a probability measure on $\mathscr{H}$, it is enough to know the joint crossing probabilities of finitely many "nice" tubes belonging to a class of tubes which is dense in $\mathcal{T}$. 
Lemma 2.11 (Characterization). Let $\hat{\mathcal{T}}$ be any dense subset of $\mathcal{T}$. Let $\mathbf{Q}_{1}$ and $\mathbf{Q}_{2}$ be two probability measures on $\mathscr{H}$ such that for all $m \geq 1$ and for all $T_{1}, \ldots, T_{m} \in \hat{\mathcal{T}}$,

$$
\mathbf{Q}_{1}\left(\boxminus_{T_{1}} \cap \cdots \cap \boxminus_{T_{m}}\right)=\mathbf{Q}_{2}\left(\boxminus_{T_{1}} \cap \cdots \cap \boxminus_{T_{m}}\right) .
$$

Then $\mathbf{Q}_{1}=\mathbf{Q}_{2}$.

PROOF. The events of the form $\boxminus_{T_{1}} \cap \cdots \cap \boxminus_{T_{m}}$ for some $m \geq 1$, and some $T_{1}, \ldots, T_{m} \in \hat{\mathcal{T}}$, form a $\pi$-system which generates the entire Borel $\sigma$-field by Theorem 2.7. By Dynkin's lemma, any two probability measures which agree on this $\pi$-system must hence be identical.

For us the main advantage of $\mathscr{H}$ being compact is that it implies that the set of all probability measures on $\mathscr{H}$ is also compact under the topology of weak convergence, hence any sequence of probability measures on $\mathscr{H}$ automatically has a subsequential limit. This greatly reduces the amount to work necessary to establish weak convergence for probability measures on $\mathscr{H}$. The following proposition gives a useful criterion for the weak convergence of a sequence of probability measures $\left(\mathbf{Q}_{\eta}\right)_{\eta>0}$ toward a limiting measure $\mathbf{Q}$ in terms of the joint crossing probabilities of certain family of tubes. But before that, we state the following definition.

A subset of tubes $\hat{\mathcal{T}} \subseteq \mathcal{T}$ is called super-dense in $\mathcal{T}$ if:

(P1) There exists a countable subset $\mathcal{T}^{0} \subseteq \mathcal{T}$ such that $\mathcal{T}^{0}$ is dense in $\mathcal{T}$.

(P2) For each $T \in \mathcal{T}^{0}$, there exists a monotone chain of tubes $T^{\delta} \in \hat{\mathcal{T}}$ indexed by $\delta \in I(T, \hat{\mathcal{T}})$, where $I(T, \hat{\mathcal{T}}) \subset[0,1]$ has a countable complement, such that $T^{0}=T$ and $T^{\delta_{1}}<T^{\delta_{2}}$ if $\delta_{1}>\delta_{2} \geq 0$ and moreover, $d_{\mathcal{T}}\left(T^{\delta}, T\right) \rightarrow 0$ as $\delta \rightarrow 0+$.

Clearly, a super-dense family of tubes is also dense in $\mathcal{T}$.

Proposition 2.12 (Convergence). Assume that $\hat{\mathcal{T}}$ is super-dense in $\mathcal{T}$. Let $\left(\mathbf{Q}_{\eta}\right)_{\eta>0}$ be a sequence of probability measures on $\mathscr{H}$. Suppose that

$$
q\left(T_{1}, \ldots, T_{m}\right):=\lim _{\eta \rightarrow 0} \mathbf{Q}_{\eta}\left(\boxminus_{T_{1}} \cap \cdots \cap \boxminus_{T_{m}}\right)
$$

exists for all $m \geq 1$ and $T_{1}, T_{2}, \ldots, T_{m} \in \hat{\mathcal{T}}$. Then there exists a (unique) probability measure $\mathbf{Q}$ on $\mathscr{H}$, such that $\mathbf{Q}_{\eta} \stackrel{d}{\rightarrow} \mathbf{Q}$. Furthermore there exists $\hat{\mathcal{T}}^{\prime} \subseteq \hat{\mathcal{T}}$ which is again super-dense in $\mathcal{T}$ and such that $\mathbf{Q}\left(\boxminus_{T_{1}} \cap \ldots \cap \boxminus_{T_{m}}\right)=q\left(T_{1}, \ldots, T_{m}\right)$ for all $T_{1}, \ldots, T_{m} \in \hat{\mathcal{T}}^{\prime}$.

Before proving the proposition, we will first note a result about the (topological) boundary of the event that a tube is traversed. If $A \subset \mathscr{H}$, let $\partial A$ denote the boundary of $A$ for the topology of $\mathscr{H}$, that is, $\partial A=\bar{A} \backslash A^{\circ}$. 
LEMMA 2.13. For $T \in \mathcal{T}$, the boundary of the closed set $\boxminus_{T}$ satisfies

$$
\partial \boxminus_{T} \subseteq\left(\bigcap_{T<T^{\prime}} \neg \boxminus_{T^{\prime}}\right) \cap \boxminus_{T} .
$$

Proof. Fix $T^{\prime}$ such that $T<T^{\prime}$. Find an open neighborhood $U$ of $T^{\prime}$ such that $T<T^{\prime \prime}$ for all $T^{\prime \prime} \in U$. Then $\boxminus^{U}$ is an open subset of $\boxminus_{T}$ and hence $\boxminus_{T^{\prime}} \subseteq$ $\boxminus^{U} \subseteq \boxminus_{T}^{\circ}$. Hence, since $\boxminus_{T}$ is closed,

$$
\partial \boxminus_{T}=\overline{\Xi_{T}} \backslash \boxminus_{T}^{\circ} \subseteq \boxminus_{T} \cap \neg \boxminus_{T^{\prime}} .
$$

The lemma follows since the above inclusion holds for all $T<T^{\prime}$.

Proof of Proposition 2.12. By compactness of $\mathscr{H}$, it suffices to establish the unique subsequential limit is $\mathbf{Q}$. Let $\mathbf{Q}^{\prime}$ be another subsequential limit of $\left(\mathbf{Q}_{\eta}\right)$. Note that by the portmanteau theorem,

$$
\mathbf{Q}\left(\boxminus_{T_{1}} \cap \cdots \cap \boxminus_{T_{m}}\right)=\lim _{\eta \rightarrow 0+} \mathbf{Q}_{\eta}\left(\boxminus_{T_{1}} \cap \cdots \cap \boxminus_{T_{m}}\right)=\mathbf{Q}^{\prime}\left(\boxminus_{T_{1}} \cap \cdots \cap \boxminus_{T_{m}}\right)
$$

for all $m \geq 1$ and $T_{1}, \ldots, T_{m} \in \hat{\mathcal{T}}$ such that $\mathbf{Q}\left(\partial \boxminus_{T_{i}}\right)=0$ and $\mathbf{Q}^{\prime}\left(\partial \boxminus_{T_{i}}\right)=0$ for each $1 \leq i \leq m$.

Let $\overline{\mathcal{T}}^{0} \subseteq \mathcal{T}$ be a countable subset satisfying the properties (P1) and (P2) in the definition of the super-dense class of tubes. By Lemma 2.13, for any two tubes $T_{1}<T_{2}$,

$$
\partial \boxminus_{T_{1}} \cap \partial \boxminus_{T_{2}}=\varnothing .
$$

Let $T \in \mathcal{T}^{0}$ and let $\left(T^{\delta}\right)_{\delta \in I(T, \hat{\mathcal{T}})} \subseteq \hat{\mathcal{T}}$ satisfy the property (P2) of super denseness. Therefore, by $\sigma$-additivity of probability measures, $\mathbf{Q}\left(\partial \boxminus_{T^{\delta}}\right)>0$ or $\mathbf{Q}^{\prime}\left(\partial \boxminus_{T^{\delta}}\right)>$ 0 can be true for only countably many $\delta \in I(T, \hat{\mathcal{T}})$. This implies that

$$
\hat{\mathcal{T}}^{\prime}:=\left\{T \in \hat{\mathcal{T}}: \mathbf{Q}\left(\partial \boxminus_{T}\right)=0, \mathbf{Q}^{\prime}\left(\partial \boxminus_{T}\right)=0\right\}
$$

is again super-dense in $\mathcal{T}$. On the other hand, for all $m \geq 1$ and for all $T_{1}, \ldots, T_{m} \in$ $\mathcal{T}^{\prime}$,

$$
\mathbf{Q}\left(\boxminus_{T_{1}} \cap \cdots \cap \boxminus_{T_{m}}\right)=\mathbf{Q}^{\prime}\left(\boxminus_{T_{1}} \cap \cdots \cap \boxminus_{T_{m}}\right)=q\left(T_{1}, \ldots, T_{m}\right) .
$$

Hence, by Lemma 2.11, $\mathbf{Q}^{\prime}=\mathbf{Q}$, as desired.

\subsubsection{Example: A super-dense family of "nice” tubes in $\mathbb{R}^{d} \times \mathbb{R}$.}

DEFINITION 2.14. Let $\mathcal{T}_{\square}$ be the family of all tubes $T$ such that:

1. The set $[T]$ can be tiled by a finite number of boxes of the form $\left[a_{1}, b_{1}\right] \times$ $\cdots \times\left[a_{d+1}, b_{d+1}\right]$ with $a_{i}<b_{i}$ for $1 \leq i \leq d+1$, and 
2. $\partial_{0} T=[T] \cap\left(\mathbb{R}^{d} \times\left\{t_{0}\right\}\right)$ and $\partial_{1} T=[T] \cap\left(\mathbb{R}^{d} \times\left\{t_{1}\right\}\right)$, where $t_{0}$ and $t_{1}$ are the start and the end time of $T$.

Let us now check that $\mathcal{T}_{\square}$ is super-dense. Define $\mathcal{T}^{0}$ same as above the definition of $\mathcal{T}_{\square}$ with an added restriction that $a_{i}$ and $b_{i}$ appearing in item 1 are all rationals. The family of tubes $\mathcal{T}^{0}$ is clearly countable and is dense in the space of tubes $\mathcal{T}$ endowed with the above metric $d_{\mathcal{T}}$. It remains to check property (P2). Let us fix a tube $T$ in $\mathcal{T}^{0}$. Suppose that

$$
\partial_{0} T=\prod_{i=1}^{d}\left[a_{i}, b_{i}\right] \times\left\{t_{-}\right\} \quad \text { and } \quad \partial_{1} T=\prod_{i=1}^{d}\left[c_{i}, d_{i}\right] \times\left\{t_{+}\right\} .
$$

By the definition of the class of tubes $\mathcal{T}_{\square}$, we can find $t_{e}>0$ such that

$$
\begin{aligned}
& {[T] \cap\left(\mathbb{R}^{d} \times\left[t_{-}, t_{-}+t_{e}\right]\right)=\prod_{i=1}^{d}\left[a_{i}, b_{i}\right] \times\left[t_{-}, t_{-}+t_{e}\right],} \\
& {[T] \cap\left(\mathbb{R}^{d} \times\left[t_{+}-t_{e}, t_{+}\right]\right)=\prod_{i=1}^{d}\left[c_{i}, d_{i}\right] \times\left[t_{+}-t_{e}, t_{+}\right] .}
\end{aligned}
$$

For $\delta<t_{e}$, define $T^{\delta}$ to be the tube such that $\left[T^{\delta}\right]=[T]^{\delta} \cap\left(\mathbb{R}^{d} \times\left[t_{-}+\delta, t_{+}-\right.\right.$ $\delta]), \partial_{0} T^{\delta}=\prod_{i=1}^{d}\left[a_{i}-\delta, b_{i}+\delta\right] \times\left\{t_{-}+\delta\right\}$ and $\partial_{1} T^{\delta}=\prod_{i=1}^{d}\left[c_{i}-\delta, d_{i}+\delta\right] \times$ $\left\{t^{+}-\delta\right\}$, where for any set $S \subseteq \mathbb{R}^{d+1}$, the enlargement $S^{\delta}$ is defined to be the closed set consisting of all points in $\mathbb{R}^{d+1}$ whose $L^{\infty}$ distance from $S$ is at most $\delta$. It is easy to check that $T^{0}=T, T^{\delta_{2}}<T^{\delta_{1}}$ if $0 \leq \delta_{1}<\delta_{2}<t_{e}$ and $d_{\mathcal{T}}\left(T^{\delta}, T\right) \rightarrow 0$ as $\delta \rightarrow 0+$. This shows the property (P2) holds.

\section{Arratia's flow in $\mathscr{H}$.}

3.1. Existence. In this section, we restrict our attention to Brownian motion on $\mathbb{R}$ and define a unique measure on $\mathscr{H}$ which represents the coalescing Brownian flow on $\mathbb{R}$ in the tube topology (for $d=1$ ). We start by introducing some notation. Let $z_{1}=\left(x_{1}, t_{1}\right), z_{2}=\left(x_{2}, t_{2}\right), \ldots$ be a sequence of space-time points in $\mathbb{R}^{2}$ and let $\mathcal{D}=\left\{z_{1}, z_{2}, \ldots\right\}$ be a countable ordered set. We assume that $\mathcal{D}$ is dense in $\mathbb{R}^{2}$. Let $\left(W_{j}(t)\right)_{t \geq t_{j}}$ be independent Brownian motions starting, respectively, from the space-time points $\left(z_{j}\right)_{j \geq 1}$, that is, $W_{j}\left(t_{j}\right)=x_{j}$, defined on a common probability space $(\Omega, \mathcal{F}, \mathbb{P})$. Using the coalescing rule as described in Section 2.5 , for each $n \geq 1$ this defines a collection of $n$ coalescing paths denoted by $W_{1}^{c}, W_{2}^{c}, \ldots, W_{n}^{c}$ starting from $z_{1}, z_{2}, \ldots, z_{n}$, respectively. This collection of coalescing paths, being finite, is of course compact in $(\Pi, \varrho)$, and hence induces, by Lemma 2.9 , a random element $\mathcal{W}_{n} \in \mathscr{H}$ defined by

$$
\mathcal{W}_{n}:=\mathcal{W}\left(z_{1}, \ldots, z_{n}\right):=\operatorname{Cr}\left(\left\{W_{1}^{c}, W_{2}^{c}, \ldots, W_{n}^{c}\right\}\right) .
$$

We now state the main theorem of the section. 
THEOREM 3.1 (Coalescing Brownian flow on $\mathbb{R}$ ). The random variables $\mathcal{W}_{n}$ converge in distribution as $n \rightarrow \infty$ to a random variable $\mathcal{W}_{\infty}$ in $\mathscr{H}$, whose law $\mathbf{P}_{\infty}$ does not depend on the dense countable set $\mathcal{D}$ (including its order).

Definition 3.2. A random variable on $\mathscr{H}$ with law $\mathbf{P}_{\infty}$ is called a coalescing Brownian flow on $\mathbb{R}$.

PROOF. By construction, $\mathcal{W}_{1} \subseteq \mathcal{W}_{2} \subseteq \cdots$ almost surely. That is, $\mathcal{W}_{k}$ is a nondecreasing sequence. Hence, by Lemma 2.8, it follows that the $\lim _{k \rightarrow \infty} \mathcal{W}_{k}$ exists almost surely in $\mathscr{H}$ and is equal to $\overline{\bigcup_{k=1}^{\infty} \mathcal{W}_{k}}$. We call $\mathcal{W}(\mathcal{D})$ the limiting element of $\mathscr{H}$ and denote by $\mathbf{P}_{\infty}^{\mathcal{D}}$ its law on $\mathscr{H}$ which, at this stage, might depend on the ordered set $\mathcal{D}$. We aim to show that the law $\mathbf{P}_{\infty}^{\mathcal{D}}$ does not in fact depend on $\mathcal{D}$.

LeMmA 3.3. Let $\mathcal{D}=\left(z_{j}\right)_{j \geq 1}$ and $\mathcal{D}^{\prime}=\left(z_{j}^{\prime}\right)_{j \geq 1}$ be two countable dense ordered sets of $\mathbb{R}^{2}$. Fix $m \geq 1$ and tubes $T_{1}, \ldots, T_{m} \in \mathcal{T}_{\square}$. Let

$$
\begin{aligned}
& p\left(T_{1}, \ldots, T_{m}\right)=\lim _{n \rightarrow \infty} \mathbb{P}\left(\mathcal{W}\left(z_{1}, \ldots, z_{n}\right) \in \boxminus_{T_{1}} \cap \cdots \cap \boxminus_{T_{m}}\right) \quad \text { and } \\
& p^{\prime}\left(T_{1}, \ldots, T_{m}\right)=\lim _{n \rightarrow \infty} \mathbb{P}\left(\mathcal{W}\left(z_{1}^{\prime}, \ldots, z_{n}^{\prime}\right) \in \boxminus_{T_{1}} \cap \cdots \cap \boxminus_{T_{m}}\right) .
\end{aligned}
$$

Then $p\left(T_{1}, \ldots, T_{m}\right)=p^{\prime}\left(T_{1}, \ldots, T_{m}\right)$.

Proof. The limits $p\left(T_{1}, \ldots, T_{m}\right)$ and $p^{\prime}\left(T_{1}, \ldots, T_{m}\right)$ exist due to monotonicity. Let $z_{j}=\left(x_{j}, t_{j}\right)$ and $z_{j}^{\prime}=\left(x_{j}^{\prime}, t_{j}^{\prime}\right)$. We may suppose without loss of generality that the points $z_{1}, z_{2}, \ldots$ (resp., $z_{1}^{\prime}, z_{2}^{\prime}, \ldots$ ) are distinct. For $1 \leq i \leq m$, let the lower face of the tube $T_{i}$ is given by $\partial_{0} T_{i}=\left[a_{i}, b_{i}\right] \times\left\{u_{i}\right\}, u_{i}$ being the start time of $T_{i}$. We denote by $A$ the event $\boxminus_{T_{1}} \cap \cdots \cap \boxminus_{T_{m}}$.

Note that a Brownian particle starting from a point in the boundary of $\left[a_{i}, b_{i}\right]$ at time $u_{i}$ will immediately escape the interval $\left[a_{i}, b_{i}\right]$ almost surely and the tube $T_{i}$ can not be crossed by it. So, as far as the event $A$ is concerned we can assume that none of the points in $z_{j}$ or $z_{j}^{\prime}$ lies in $\left\{\left(a_{i}, u_{i}\right),\left(b_{i}, u_{i}\right): 1 \leq i \leq n\right\}$, the boundary of the lower face of any tube.

For $x, t \in \mathbb{R}$ and $\varepsilon>0$, define two rectangles $R(x, t ; \varepsilon)=[x-\varepsilon, x+\varepsilon] \times[t-$ $\varepsilon, t+\varepsilon]$ and $R^{+}(x, t ; \varepsilon)=[x-\varepsilon, x+\varepsilon] \times[t, t+\varepsilon]$. Let $W$ be Brownian motion starting from the space-time point $(x, t)$ and $W^{\prime}$ be another independent Brownian motion starting from the space-time point $\left(x^{\prime}, t^{\prime}\right)$. Let $\tau \geq \max \left(t, t^{\prime}\right)$ be the first hitting time of $W$ and $W^{\prime}$. Then for any fixed $\varepsilon>0$, the probability of the event

$$
\begin{array}{r}
\{\tau<t+\varepsilon \text { and }(W(s), s) \in R(x, t ; \varepsilon) \forall t \leq s \leq \tau \text { and } \\
\left.\left(W^{\prime}(s), s\right) \in R(x, t ; \varepsilon) \forall t^{\prime} \leq s \leq \tau\right\}
\end{array}
$$

converges to 1 as $\left(x^{\prime}, t^{\prime}\right) \rightarrow(x, t)$. This follows from the right continuity of Brownian paths and the fact that $\tau \searrow t$ almost surely as $\left(x^{\prime}, t^{\prime}\right) \rightarrow(x, t)$.

Fix $n \in \mathbb{N}$ and $\delta>0$. We can choose $\varepsilon_{0}>0$ sufficiently small such that it satisfies: 
(i) $R\left(x_{j}, t_{j}, \varepsilon_{0}\right), 1 \leq j \leq n$ are pairwise disjoint.

(ii) For each $1 \leq j \leq n$ and each $1 \leq i \leq m$, if $t_{j}<u_{i}$ then $t_{j}+\varepsilon_{0}<u_{i}$.

(iii) For each $1 \leq j \leq n$ and each $1 \leq i \leq m$, if $t_{j}=u_{i}$ and $x_{j} \in\left(a_{i}, b_{i}\right)$, then $R^{+}\left(x_{j}, t_{j}, \varepsilon_{0}\right) \subseteq\left[T_{i}\right]$.

Given any $\delta>0$ and some $\varepsilon>0$ that will be specified in a moment, find $y_{1}^{\prime}=$ $\left(v_{1}^{\prime}, s_{1}^{\prime}\right), \ldots, y_{n}^{\prime}=\left(v_{n}^{\prime}, s_{n}^{\prime}\right) \in \mathcal{D}^{\prime}$ such that $s_{j}^{\prime} \leq t_{j}$ and $\left|x_{j}-v_{j}^{\prime}\right|+\left|t_{j}-s_{j}^{\prime}\right| \leq \varepsilon$ for $1 \leq j \leq n$. This is possible since $\mathcal{D}^{\prime}$ is dense in $\mathbb{R}^{2}$. By (3.2), there is $\varepsilon_{0}$ such that if we choose $\varepsilon<\varepsilon_{0}$ sufficiently small, then for each $1 \leq j \leq n$, two independent Brownian motions starting from the space-time points $z_{j}$ and $y_{j}^{\prime}$ collide before the graphs of their trajectories leave the rectangle $R\left(x_{j}, t_{j}, \varepsilon_{0}\right)$ with probability at least $1-\frac{\delta}{n}$. Obviously,

$$
\mathbb{P}\left(\mathcal{W}\left(z_{1}, \ldots, z_{n}\right) \in A\right) \leq \mathbb{P}\left(\mathcal{W}\left(y_{1}^{\prime}, \ldots, y_{n}^{\prime}, z_{1}, \ldots, z_{n}\right) \in A\right),
$$

by invariance under reordering (using the strong Markov property of Brownian motion). Thus, by our choice of $\varepsilon$ and a simple union bound, we obtain

$$
\begin{aligned}
& \mathbb{P}\left(\mathcal{W}\left(y_{1}^{\prime}, \ldots, y_{n}^{\prime}, z_{1}, \ldots, z_{n}\right) \in A\right) \\
& \quad \leq \mathbb{P}\left(\mathcal{W}\left(y_{1}^{\prime}, \ldots, y_{n}^{\prime}\right) \in A\right)+\delta \\
& \quad \leq p^{\prime}\left(T_{1}, \ldots, T_{m}\right)+\delta .
\end{aligned}
$$

Combining (3.3) and (3.4), we obtain that, for each $n \geq 1$

$$
\mathbb{P}\left(\mathcal{W}\left(z_{1}, \ldots, z_{n}\right) \in A\right) \leq p^{\prime}\left(T_{1}, \ldots, T_{m}\right)+\delta .
$$

Taking limit as $n \rightarrow \infty$ and noting that $\delta>0$ is arbitrary, we have $p\left(T_{1}, \ldots, T_{m}\right) \leq$ $p^{\prime}\left(T_{1}, \ldots, T_{m}\right)$. Interchanging the role of $\mathcal{D}$ and $\mathcal{D}^{\prime}$ we deduce the equality. This completes the proof of the lemma.

Now, let us show how Lemma 3.3 implies Theorem 3.1. Take any countable dense set $\mathcal{D}^{\prime}=\left(z_{j}^{\prime}\right)_{j \geq 1}$. By Lemma 3.3, for fixed tubes $T_{1}, \ldots, T_{m} \in \mathcal{T}_{\square}$,

$$
\begin{aligned}
p\left(T_{1}, \ldots, T_{m}\right) & =\lim _{n \rightarrow \infty} \mathbb{P}\left(\mathcal{W}\left(z_{1}, \ldots, z_{n}\right) \in A\right)=\lim _{n \rightarrow \infty} \mathbb{P}\left(\mathcal{W}\left(z_{1}^{\prime}, \ldots, z_{n}^{\prime}\right) \in A\right) \\
& =p^{\prime}\left(T_{1}, \ldots, T_{m}\right) .
\end{aligned}
$$

Since $\mathcal{T}_{\square}$ is super-dense in $\mathcal{T}$, by Proposition 2.12, we know that $p\left(T_{1}, \ldots, T_{m}\right)=$ $\mathbf{P}_{\infty}^{\mathcal{D}}(A)$ and $p^{\prime}\left(T_{1}, \ldots, T_{m}\right)=\mathbf{P}_{\infty}^{\mathcal{D}^{\prime}}(A)$ at least when $T_{1}, \ldots, T_{m} \in \mathcal{T}^{\prime}$, where $\mathcal{T}^{\prime}$ is super-dense in $\mathcal{T}$. Thus, we conclude $\mathbf{P}_{\infty}^{\mathcal{D}}(A)=\mathbf{P}_{\infty}^{\mathcal{D}^{\prime}}(A)$ if $T_{1}, \ldots, T_{m} \in \mathcal{T}^{\prime}$. By Lemma 2.11, this shows $\mathbf{P}_{\infty}^{\mathcal{D}}=\mathbf{P}_{\infty}^{\mathcal{D}^{\prime}}$ and completes the proof of Theorem 3.1. 
3.2. Characterization of Arratia's flow. Let $\mathbf{P}_{\infty}$ be the law of $\mathcal{W}_{\infty}$ on $\mathscr{H}$, and for any tubes $T_{1}, \ldots, T_{m} \in \mathcal{T}$ (and hence in $\mathcal{T}_{\square}$ ), let

$$
p\left(T_{1}, \ldots, T_{m}\right)=\lim _{n \rightarrow \infty} \mathbb{P}\left(\mathcal{W}\left(z_{1}, \ldots, z_{n}\right) \in \boxminus_{T_{1}} \cap \cdots \cap \boxminus_{T_{m}}\right) .
$$

It follows from Theorem 3.1 and Proposition 2.12 that there exists a super-dense family of tubes $\mathcal{T}_{\square}^{\prime}$ such that for all $T_{1}, \ldots, T_{m} \in \mathcal{T}_{\square}^{\prime}$,

$$
p\left(T_{1}, \ldots, T_{m}\right)=\mathbf{P}_{\infty}\left(\boxminus_{T_{1}} \cap \cdots \cap \boxminus_{T_{m}}\right) .
$$

This characterises uniquely $\mathbf{P}_{\infty}$, though in practice a drawback of this conclusion is that we do not know what $\mathcal{T}_{\square}^{\prime}$ is. However, the following result shows that this conclusion remains valid for all $T_{1}, \ldots, T_{m} \in \mathcal{T}_{\square}$. We stress, however, that this result is not needed for the rest of the paper, so this section may be skipped by a reader who is only interested in the invariance principle (Theorem 4.1).

THEOREM 3.4. $\quad \mathbf{P}_{\infty}$ is the unique probability distribution on $\mathscr{H}$ such that for all $m \geq 1$ and for all fixed tubes $T_{1}, \ldots, T_{m} \in \mathcal{T}_{\square}$,

$$
\begin{aligned}
& \mathbf{P}_{\infty}\left(\boxminus_{T_{1}} \cap \cdots \cap \boxminus_{T_{m}}\right) \\
& \quad=\sup _{n \geq 1 ; z_{1}, \ldots, z_{n} \in \mathbb{R}^{2}} \mathbb{P}\left(\mathcal{W}\left(z_{1}, \ldots, z_{n}\right) \in \boxminus_{T_{1}} \cap \cdots \cap \boxminus_{T_{m}}\right) .
\end{aligned}
$$

PROOF. Note that the supremum in the right-hand side of (3.6) is simply $p\left(T_{1}, \ldots, T_{m}\right)$. The lower bound is easy: indeed, since $A=\boxminus_{T_{1}} \cap \cdots \cap \boxminus_{T_{m}}$ is closed in $\mathscr{H}$, and since $\mathcal{W}\left(z_{1}, \ldots, z_{n}\right) \rightarrow \mathcal{W}_{\infty}$ in distribution on $\mathscr{H}$,

$$
\mathbf{P}_{\infty}(A) \geq \lim _{n \rightarrow \infty} \mathbb{P}\left(\mathcal{W}\left(z_{1}, \ldots, z_{n}\right) \in A\right) .
$$

Taking a supremum over $n \geq 1$ and $z_{1}, \ldots, z_{n}$ shows that

$$
\mathbf{P}_{\infty}(A) \geq \sup _{n \geq 1 ; z_{1}, \ldots, z_{n} \in \mathbb{R}^{2}} \mathbb{P}\left(\mathcal{W}\left(z_{1}, \ldots, z_{n}\right) \in A\right) .
$$

We now turn to proof of the upper bound in (3.6). Recall that by Lemma 2.8, $\mathbf{P}_{\infty}^{\mathcal{D}}(A)=\mathbb{P}\left(T_{1}, \ldots, T_{m} \in \overline{\bigcup_{k} \mathcal{W}_{k}}\right)$, where $A=\boxminus_{T_{1}} \cap \cdots \cap \boxminus_{T_{m}}$. We need to prove that for any finite number of tubes $T_{1}, T_{2}, \ldots, T_{m} \in \mathcal{T}_{\square}$,

$$
\mathbb{P}\left(T_{1}, \ldots, T_{m} \in \overline{\bigcup_{k} \mathcal{W}_{k}}\right)=\mathbb{P}\left(T_{1}, \ldots, T_{m} \in \bigcup_{k} \mathcal{W}_{k}\right),
$$

as the right-hand side of the above equation is the increasing limit (as $k \rightarrow \infty$ ) of

$$
\mathbb{P}\left(\mathcal{W}\left(z_{1}, \ldots, z_{k}\right) \in \boxminus_{T_{1}} \cap \cdots \cap \boxminus_{T_{m}}\right) .
$$

Note that it is enough to prove the above equality for $m=1$ since

$$
\begin{gathered}
\mathbb{P}\left(T_{1}, \ldots, T_{m} \in \overline{\bigcup_{k} \mathcal{W}_{k}}\right)-\mathbb{P}\left(T_{1}, \ldots, T_{m} \in \bigcup_{k} \mathcal{W}_{k}\right) \\
\leq \sum_{i=1}^{m}\left(\mathbb{P}\left(T_{i} \in \overline{\bigcup_{k} \mathcal{W}_{k}}\right)-\mathbb{P}\left(T_{i} \in \mathcal{W}_{k}\right)\right) .
\end{gathered}
$$


So, we fix a tube $T \in \mathcal{T}_{\square}$. We are going to show that

$$
\mathbb{P}\left(T \in \bigcup_{k} \mathcal{W}_{k}\right)=\mathbb{P}\left(T \in \overline{\bigcup_{k} \mathcal{W}_{k}}\right) .
$$

Now $T \in \overline{\bigcup_{k} \mathcal{W}_{k}}$ means that there exists an increasing sequence $k_{n}$ of integers and tubes $T_{n} \in \mathcal{W}_{k_{n}}$ such that $T_{n} \rightarrow T$. For any tube $T^{\prime}<T$, we can always find an open neighborhood $U$ of $T$ such that $T^{\prime}<T^{\prime \prime}$ for all $T^{\prime \prime} \in U$. Consequently, $T^{\prime}<T_{n}$ for large enough $n$ and thus $T^{\prime} \in \mathcal{W}_{k_{n}} \subseteq \bigcup_{k} \mathcal{W}_{k}$. This implies that for any tube $T^{\prime}<T$, we have

$$
\mathbb{P}\left(T \in \overline{\bigcup_{k} \mathcal{W}_{k}}\right) \leq \mathbb{P}\left(T^{\prime} \in \bigcup_{k} \mathcal{W}_{k}\right) .
$$

Our goal is to show that for any $\varepsilon>0$, there exists a tube $T^{\prime} \in \mathcal{T}$, such that $T^{\prime}<T$ and

$$
\mathbb{P}\left(T^{\prime} \in \bigcup_{k} \mathcal{W}_{k}\right) \leq \mathbb{P}\left(T \in \bigcup_{k} \mathcal{W}_{k}\right)+\varepsilon,
$$

which immediately implies (3.7). Let $\partial_{0} T=[a, b] \times\left\{t_{0}\right\}$ and $\partial_{1} T=[c, d] \times\left\{t^{*}\right\}$. By the definition of the class of tubes $\mathcal{T}_{\square}$, we can find $t_{e}>0$ such that $[T] \cap(\mathbb{R} \times$ $\left.\left[t_{0}, t_{0}+t_{e}\right]\right)=[a, b] \times\left[t_{0}, t_{0}+t_{e}\right]$ and $[T] \cap\left(\mathbb{R} \times\left[t^{*}-t_{e}, t^{*}\right]\right)=[c, d] \times\left[t^{*}-\right.$ $\left.t_{e}, t^{*}\right]$.

For $\delta<t_{e}$, recall the tube $T^{\delta} \in \mathcal{T}_{\square}$ introduced in Section 2.6.1: this is the tube such that $\left[T^{\delta}\right]=[T]^{\delta} \cap\left(\mathbb{R} \times\left[t_{0}+\delta, t^{*}-\delta\right]\right), \partial_{0} T^{\delta}=[a-\delta, b+\delta] \times\left\{t_{0}+\delta\right\}$ and $\partial_{1} T^{\delta}=[c-\delta, d+\delta] \times\left\{t^{*}-\delta\right\}$. It follows from the definition of the class of tubes $\hat{\mathcal{T}}$ that $T^{\delta}<T$ and $d_{\mathcal{T}}\left(T^{\delta}, T\right) \rightarrow 0$ as $\delta \rightarrow 0+$.

We are going to prove (3.8) taking $T^{\prime}=T^{\delta}$ for some small enough $\delta$, depending only on $\varepsilon$ and $T \in \mathcal{T}_{\square}$. We are going to argue this by a (rather long) series of simple observations.

For $s<t$ and an interval $I$, let $A_{I}(s, t)$ denote the locations of particles at time $t$ whose trajectories started before or at time $s$ and stayed in $I$ throughout $[s, t]$. The proof of Lemma 3.3 also shows that the law of $A_{I}(s, t)$ does not depend on $\mathcal{D}$. We first need a well-known and simple lemma which states that the coalescing Brownian paths "come down from infinity".

LEMMA 3.5. If I is bounded then $\left|A_{I}(s, t)\right|<\infty$ almost surely for $s<t$. Its law depends only on $t-s$ and I, and is continuous (say in total variation) in both $s$ and $t$ if $s<t$.

PROOF. The first statement is well known and follows, for instance, from Arratia's work [4]. The continuity of the law of $\left|A_{I}(s, t)\right|$, in total variation, is trivial to verify as $s$ is fixed and $t$ varies, since a.s. $\lim _{\eta \rightarrow 0}\left|A_{I}(s, t+\eta)\right|=\left|A_{I}(s, t)\right|$. Hence, continuity follows in both $s$ and $t$ provided that $s<t$. 
Now let $\alpha=\frac{t_{e}}{4}$ be fixed. Set $I=[a, b]$ and $I^{\delta}=[a-\delta, b+\delta]$, where $\delta$ will be chosen sufficiently small. Let $t_{1}=t_{0}+\delta$ and $t_{2}=t_{0}+\delta+\alpha$. Consider the sets $A_{0}=A_{I}\left(t_{0}, t_{2}\right)$ and $A_{1}=A_{I^{\delta}}\left(t_{1}, t_{2}\right)$.

Lemma 3.6. For $\varepsilon>0$, we can choose $\delta_{0}>0$ so that $A_{0}$ and $A_{1}$ agree with probability greater than $1-\varepsilon$ for all $\delta<\delta_{0}$.

Proof. Note that $A_{0} \subset A_{1}$, and hence it suffices to show that $\mathbb{P}\left(\left|A_{0}\right|=\right.$ $\left.\left|A_{1}\right|\right) \geq 1-\varepsilon$. It is a straightforward consequence of Lemma 3.5 that $\left|A_{I}\left(t_{1}, t_{2}\right)\right|$ and $\left|A_{I}\left(t_{0}, t_{2}\right)\right|$ agree with probability greater than $1-\varepsilon / 2$ for $\delta$ sufficiently small. Using scale and translation invariance of Brownian motion and the same argument, we see also that $\left|A_{I}\left(t_{1}, t_{2}\right)\right|$ and $\left|A_{I^{\delta}}\left(t_{1}, t_{2}\right)\right|$ agree with probability greater than $1-\varepsilon / 2$. Hence, the result follows.

Now for $\kappa>0$, define $J=[a+\kappa, b-\kappa]$.

LEMMA 3.7. For all $\varepsilon>0$, we can choose $\kappa>0$ and $\delta_{0}>0$ so that, uniformly in $\delta<\delta_{0}$, the tube $T^{\delta}$ is crossed if and only if it is crossed by a path touching $\left(A_{1} \cap J\right) \times\left\{t_{2}\right\}$, except with probability at most $\varepsilon$.

PROOF. Obviously, if $T^{\delta}$ is crossed by a particle, then that particle has to touch $A_{1} \times\left\{t_{2}\right\}$. So, it suffices to show that with probability at least $1-\varepsilon$, none of the particles starting from $\left(A_{1} \cap J^{c}\right) \times\left\{t_{2}\right\}$ will stay inside $\left[T^{\delta}\right]$ up to time $t_{0}+\frac{t_{e}}{2}$.

Clearly, $I^{\delta} \backslash J \subset[a-\kappa, a+\kappa] \cup[b-\kappa, b+\kappa]$ if $\delta<\kappa$. Now, choose $n$ large enough that for all $\delta<\frac{\alpha}{2},\left|A_{1}\right| \leq\left|A_{[a-1, b+1]}\left(t_{0}+\frac{\alpha}{2}, t_{0}+\alpha\right)\right| \leq n$ with probability greater than $1-\frac{\varepsilon}{3}$. Observe that we can take $\kappa>0$ small enough such that any coalescing Brownian path in $[a-\kappa, a+\kappa]$ at time $t_{2}$ will hit the line $x=a-\kappa$ by time $t_{2}+\frac{t_{e}}{4}$ with probability at least $1-\frac{\varepsilon}{3 n}$, uniformly in $\delta$. Such a particle necessarily leaves $T$ and $T^{\delta}$, if $\delta<\kappa$. Likewise, any coalescing Brownian path in $[b-\kappa, b+\kappa]$ at $t_{2}$ will also hit the line $y=b+\kappa$ by time $t_{2}+\frac{t_{e}}{4}$ with probability at least $1-\frac{\varepsilon}{3 n}$, uniformly in $\delta$. Summing over all particles at time $t_{2}$, we see that with probability greater than $1-\varepsilon$, any particle at time $t_{2}$ located within $\left(I^{\delta} \backslash J\right) \cap A_{1}$ can cross $T^{\delta}$. Lemma 3.7 follows with $\delta_{0}=\min \left(\kappa, \frac{\alpha}{2}\right)$.

Combining Lemmas 3.6 and 3.7, we deduce that for $\delta<\delta_{0}$ (with $\delta_{0}$ as in Lemmas 3.6 and 3.7, depending only on $\varepsilon$ and $T$ ),

$$
\mathbb{P}\left(T^{\delta} \in \bigcup_{k} \mathcal{W}_{k}\right)-\mathbb{P}\left(T \in \bigcup_{k} \mathcal{W}_{k}\right) \leq 2 \varepsilon+p,
$$

where $p$ is the probability that one of the particles passing through $\left(x, t_{2}\right)$ for some $x \in A_{1} \cap J$ will stay within $\left[T^{\delta}\right]$ until the time $t^{*}-\delta$ but the trajectory of that particle will leave the tube $T$ at sometime between $t_{2}$ and $t^{*}$. Recall also that, by Lemma 3.5, we can find a large $n$ (depending only on $\varepsilon$ and $T$ ) such that $\left|A_{1}\right| \leq n$ 


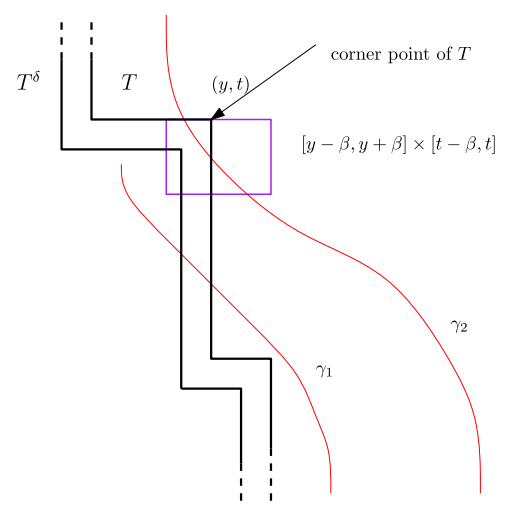

FIG. 3. The path $\gamma_{1}$ leaves both $T$ and $T^{\delta}$ whereas the path $\gamma_{2}$ exits from $T$ near a corner point and continues to stay inside $T^{\delta}$.

with probability at least $1-\varepsilon$. Hence, using the Markov property of Brownian motion, we complete the proof of (3.8) using the following lemma.

LEMMA 3.8. Let $B_{\delta, x}$ be the event that a Brownian motion starting from $x$ at time $t_{2}$ will stay within $T^{\delta}$ until $t^{*}-\delta$ but the trajectory of that Brownian motion leaves the tube $T$ at sometime between $t_{2}$ and $t^{*}$. Then

$$
\limsup _{\delta \rightarrow 0+} \sup _{x \in[a+\kappa, b-\kappa]} \mathbb{P}\left(B_{\delta, x}\right)=0 .
$$

Proof. $\quad$ Note that if $T \in \mathcal{T}_{\square}$, then the boundary of the set [ $T$ ] can be expressed as the union of finitely many vertical and horizontal line segments. We call a point $(y, t)$ a corner point of $T$ if $(y, t)$ lies at the point of intersection of a vertical and a horizontal line segment on the boundary of $[T]$ as described in Figure 3 above. Let $S$ be the set of all corner points $(y, t)$ of $T$ such that $t_{2}<t \leq t^{*}$. Let $W^{x}$ be Brownian motion starting from $x$ at time $t_{2}$.

To estimate $\mathbb{P}\left(B_{\delta, x}\right)$, we consider the following event:

$$
W^{x}(s) \in[y-\beta, y+\beta] \quad \text { for some } s \in[t-\beta, t] \text { and }(y, t) \in S .
$$

Since the set $S$ is finite, we can find $\beta>0$ small such that the above event has probability at most $\varepsilon$, uniformly in $x \in[a+\kappa, b-\kappa]$.

Note that if the above event does not occur and if $\delta \in(0, \beta)$, then for the event $B_{\delta, x}$ to happen, $W^{x}$ must exit the tube $T$ for the first time through some point $\left(y^{\prime}, t^{\prime}\right)$ on the left or the right boundary of $[T]$ such that:

(i) the vertical line segment joining the points $\left(y^{\prime}, t^{\prime}\right)$ and $\left(y^{\prime}, t^{\prime}+\beta\right)$ lies on the boundary of $[T]$ and

(ii) for all time $\left[t^{\prime}, t^{\prime}+\beta\right]$, the trajectory of $W^{x}$ must continue to stay inside the lines $x=y^{\prime}-\delta$ or $x=y^{\prime}+\delta$, depending on which of the two boundaries (left 
or right) of $T$ it violates. By choosing $\delta>0$ small, we can make this probability smaller than $\varepsilon$. Thus, for small enough $\delta>0$,

$$
\sup _{x \in[a+\kappa, b-\kappa]} \mathbb{P}\left(B_{\delta, x}\right) \leq 2 \varepsilon,
$$

which proves (3.9). This establishes (3.8), and thus completes the proof of the upper bound.

In turn, this completes the proof of Theorem 3.4.

4. Invariance principle for coalescing random walks. Consider a system of independent coalescing random walks started from every space-time point $(x, t)$ on $\mathbb{Z} \times \mathbb{Z}$. We assume that the step distribution $\xi$ satisfies

$$
\mathbb{E}[\xi]=0, \quad \mathbb{E}\left[\xi^{2}\right]=\sigma^{2} \text { and } \xi \text { is aperiodic. }
$$

Under diffusive scaling, this gives rise to a collection of continuous paths in $(\Pi, \varrho)$ obtained by interpolating linearly the paths of the coalescing random walk in the rescaled lattice $\mathbb{L}_{\eta}:=\sigma^{-1} \eta \mathbb{Z} \times \eta^{2} \mathbb{Z}$, which we will denote by $\Gamma^{\eta}$. Note that in $\Gamma^{\eta}$, two paths can cross over each other several times before they finally merge at some point in $\mathbb{L}_{\eta}$. Note that if $\mathcal{W}^{\eta}:=\operatorname{Cr}\left(\Gamma^{\eta}\right)$ then $\mathcal{W}^{\eta} \in \mathscr{H}$ by Remark 2.10: indeed, in the notation of this remark, the collection of paths $\left(\Gamma^{\eta}\right)_{T}$, restricted to any particular tube $T$, is finite, and hence compact. We call $\mathbf{P}^{\eta}$ the law on $\mathscr{H}$ of $\mathcal{W}^{\eta}$.

THEOREM 4.1. Assume (4.1). Then as $\eta \rightarrow 0$,

$$
\mathbf{P}^{\eta} \rightarrow \mathbf{P}_{\infty}
$$

weakly in $\mathscr{H}$, where $\mathbf{P}_{\infty}$ is the law of the coalescing Brownian flow on $\mathscr{H}$, as defined in Theorem 3.1.

REMARK 4.2. As can be trivially seen from the proof, the same conclusion holds for many variants. Here is one such example. Fix a step distribution $\xi$ which is nonlattice, centred and such that $\mathbb{E}[\xi]=0$ and $\mathbb{E}\left[\xi^{2}\right]=\sigma^{2}$. Consider $\Gamma^{\eta}$ a system of coalescing random walks in continuous time (jumping at rate one according to the distribution $\xi$ ). The particles start from a cloud of points $(x, t) \in \mathbb{R} \times \mathbb{R}$ distributed according to a Poisson point process with intensity $d x \otimes d t$, and particles coalesce as soon as their mutual distance is less than one (i.e., the path of the particle with higher label merges with the path of the particle with lower label, in some fixed enumeration of the Poisson cloud particles, as in Section 3.1). Then applying the same diffusive scaling, this gives rise to a law $\mathbf{P}^{\eta}$ on $\mathscr{H}$ which converges weakly to Arratia's flow $\mathbf{P}_{\infty}$ as $\eta \rightarrow 0$.

Proof of TheOREM 4.1. Fix $T_{1}, \ldots, T_{m} \in \mathcal{T}_{\square}$, and recall our notation from equation (3.1) and Lemma 3.3:

$$
p\left(T_{1}, \ldots, T_{m}\right)=\lim _{n \rightarrow \infty} \mathbb{P}\left(\mathcal{W}_{n} \in A\right),
$$


where $A=\boxminus_{T_{1}} \cap \cdots \cap \boxminus_{T_{m}}$. We split the proof of Theorem 4.1 into two parts, a lower and an upper bound. The lower bound will consist in showing that for $T_{1}, \ldots, T_{m} \in \mathcal{T}_{\square}$

$$
\liminf _{\eta \rightarrow 0} \mathbf{P}_{\eta}(A) \geq p\left(T_{1}, \ldots, T_{m}\right) .
$$

For the upper bound, we will show that for $T_{1}, \ldots, T_{m} \in \mathcal{T}_{\square}$,

$$
\limsup _{\eta \rightarrow 0} \mathbf{P}_{\eta}(A) \leq \mathbf{P}_{\infty}(A) \text {. }
$$

By Proposition 2.12, there exists a super-dense family $\mathcal{T}_{\square}^{\prime}$ such that when $T_{1}, \ldots, T_{m} \in \mathcal{T}_{\square}^{\prime}$, it holds that $p\left(T_{1}, \ldots, T_{m}\right)=\mathbf{P}_{\infty}(A)$. Consequently, by (4.2) and (4.3), if $T_{1}, \ldots, T_{m} \in \mathcal{T}_{\square}^{\prime}$, then

$$
\lim _{\eta \rightarrow 0} \mathbf{P}_{\eta}(A)=\mathbf{P}_{\infty}(A)
$$

Since $\mathcal{T}_{\square}^{\prime}$ is itself super-dense, another application of Proposition 2.12 completes the proof of the theorem.

4.1. Proof of lower bound. Fix $n \geq 1$ and let $z_{1}, \ldots, z_{n} \in \mathbb{R}^{2}$. Let $z_{1}^{\eta}, \ldots, z_{n}^{\eta}$ be space-time points in the rescaled lattice $\mathbb{L}_{\eta}$ such that $z_{1}^{\eta} \rightarrow z_{1}, \ldots, z_{n}^{\eta} \rightarrow z_{n}$ as $\eta \rightarrow 0$. Let $\Gamma_{n}^{\eta}=\Gamma^{\eta}\left(z_{1}^{\eta}, \ldots, z_{n}^{\eta}\right)$ be a system of $n$ independent rescaled coalescing random walks in $\mathbb{L}_{\eta}$ started from $z_{1}^{\eta}, \ldots, z_{n}^{\eta}$, viewed as a random element of $\left(\Pi^{n}, \varrho^{n}\right)$ as defined in (2.5). Let $\Gamma_{n}=\Gamma\left(z_{1}, \ldots, z_{n}\right)$ be a system of $n$ independent coalescing Brownian motions started from $z_{1}, \ldots, z_{n}$, also viewed as a random variable in $\left(\Pi^{n}, \varrho^{n}\right)$.

LEMMA 4.3. As $\eta \rightarrow 0$,

$$
\Gamma_{n}^{\eta} \rightarrow \Gamma_{n}
$$

in distribution on $\left(\Pi^{n}, \varrho^{n}\right)$.

The lemma says that $n$ coalescing random walks converge to $n$ coalescing Brownian motions, which is of course hardly surprising. For a detailed proof of this fact, see [20], which we will not repeat here. But later while treating coalescing random walks on Sierpinski gasket, we will provide a new proof of the above result that holds in greater generality.

The proof of the lower bound (4.2) is now easy. Fix $n \geq 1$ and $z_{1}, \ldots, z_{n} \in \mathbb{R}^{2}$. Let $z_{1}^{\eta}, \ldots, z_{n}^{\eta} \in \mathbb{L}_{\eta}$ such that $z_{i}^{\eta} \rightarrow z_{i}$. Let $\mathcal{W}_{n}^{\eta}=\operatorname{Cr}\left(\Gamma^{\eta}\left(z_{1}^{\eta}, \ldots, z_{n}^{\eta}\right)\right)$ and $\mathcal{W}_{n}=$ $\operatorname{Cr}\left(\Gamma\left(z_{1}, \ldots, z_{n}\right)\right)$. By monotonicity,

$$
\begin{aligned}
\mathbf{P}^{\eta}\left(\boxminus_{T_{1}} \cap \cdots \cap \boxminus_{T_{m}}\right) & \geq \mathbb{P}\left(\mathcal{W}_{n}^{\eta} \in \boxminus_{T_{1}} \cap \cdots \cap \boxminus_{T_{m}}\right) \\
& \rightarrow \mathbb{P}\left(\mathcal{W}_{n} \in \boxminus_{T_{1}} \cap \cdots \cap \boxminus_{T_{m}}\right)
\end{aligned}
$$


as $\eta \rightarrow 0$, where the convergence follows from Lemma 4.3. Taking a limit as $n \rightarrow$ $\infty$, for a fixed enumeration $z_{1}, z_{2}, \ldots$ of a dense countable set $\mathcal{D}$ in $\mathbb{R}^{2}$, we obtain by Theorem 3.1

$$
\liminf _{\eta \rightarrow 0} \mathbf{P}^{\eta}\left(\boxminus_{T_{1}} \cap \cdots \cap \boxminus_{T_{m}}\right) \geq p\left(T_{1}, \ldots, T_{m}\right),
$$

as desired.

4.2. Uniform coming down from infinity. Let us now prove (4.3), which, together with (4.2) proves Theorem 4.1, as already explained. The proof of (4.3) relies essentially on the following property, which we call uniform coming down from infinity. It says that, after a positive amount of time $\delta>0$ the number of rescaled coalescing random walks which are killed upon exiting a bounded region of space stays finite as $\eta \rightarrow 0$ (i.e., is a tight family of random variables).

Proposition 4.4. Let $K>0$ be fixed. Consider coalescing random walks on $\mathbb{Z} \times \mathbb{Z}$ with increments distributed as $\xi$ satisfying (4.1), starting from each $x \in[-K n, K n] \cap \mathbb{Z}$ at time 0 , and that are killed upon leaving the interval $[-K n, K n]$. For $\delta>0$, let $U_{n}$ be the number of distinct coalescing random walks at time $\delta n^{2}$. Then there exists a constant $C$ independent of $\delta$ and $n$ such that for all $k, n \in \mathbb{Z}_{+}$,

$$
\mathbb{P}\left(U_{n} \geq k\right) \leq \frac{C}{\delta k}
$$

We start with a simple lemma.

LEMMA 4.5. Let $X^{1}, X^{2}$ be two independent random walks on $\mathbb{Z}$ with increments distributed as $\xi$ and starting at $x, y \in \mathbb{Z}$ at time 0 , respectively. Let $\tau_{x, y}$ be the integer stopping time when the two walkers first meet. Then for all $t \in \mathbb{Z}_{+}$,

$$
\mathbb{P}\left(\tau_{x, y}>t\right) \leq \frac{C_{0}}{\sqrt{t}}|x-y|
$$

for some constant $C_{0}$ independent of $t, x$ and $y$.

PROOF. This bound is easy to derive and had already been used by [20] (see Lemma 2.2). Assume without loss of generality that $x<y$. When $|x-y|=1$, this is simply Proposition 32.4 in [24]. When $|x-y|>1$, imagine that there are coalescing random walks started at every position in $\{x, x+1, \ldots, y\}$. Until time $\tau_{x, y}$, we may regard $X^{1}$ as the path started from $x$ and $X^{2}$ as the path started from $y$. If all the random walks have coalesced by time $t$, then $X^{1}$ and $X^{2}$ have also coalesced, and hence $\tau_{x, y} \leq t$. Thus,

$$
\mathbb{P}\left(\tau_{x, y}>t\right) \leq \sum_{i=x}^{y-1} \mathbb{P}\left(\tau_{i, i+1}>t\right) \leq \frac{C_{0}}{\sqrt{t}}|x-y|
$$

by the case $|x-y|=1$. 
Proof of Proposition 4.4. Suppose there are $m$ distinct particles in the interval $[-K n, K n]$ at time 0 . By the pigeonhole principle, there exists at least one pair of particles that are at a distance of at most $a_{m, n}:=2 \mathrm{Kn} / \mathrm{m}$. By Lemma 4.5, the probability that these two unkilled random walkers will meet each other by time $t_{m, n}:=4 C_{0}^{2} a_{m, n}^{2}$ is at least $1 / 2$. Hence, in the coalescing system the probability that there is at most $m-1$ particles after time $t_{m, n}$ is certainly at least $1 / 2$. This happens due to one of the following scenarios:

(i) At least one of the particles leaves the interval $[-K n, K n]$ and hence gets killed.

(ii) The two distinguished particles collide with each other and no others.

(iii) Some other particle(s) collides with one or both of the distinguished particles.

Moreover, if after time $t_{m, n}$, the number of distinct particles in the coalescing system still remains $m$ then we can again find at time $t_{m, n}$ a possibly different pair of particles that are within distance $a_{m, n}$ from each other, and the probability that this pair of particles will collide within the time interval $\left[t_{m, n}, 2 t_{m, n}\right]$ is again at least $1 / 2$. By repeating this argument and using the Markov property, we see that if we let $\tau_{m-1}^{m}=\tau_{m-1}^{m}(n)$ be the first time there are $m-1$ surviving particles starting from $m$ particles, then, regardless of the particular initial configuration of the $m$ particles in $[-K n, K n]$,

$$
\mathbb{P}\left(\tau_{m-1}^{m} \geq k t_{m, n}\right) \leq 2^{-k} .
$$

In particular, $\mathbb{E}\left[\tau_{m-1}^{m}\right] \leq 2 t_{m, n}$. Thus, if we start with one particle at each $x \in$ $[-K n, K n] \cap \mathbb{Z}$, then the probability that after $\delta n^{2}$ time the number of particles remaining is greater than $k$ is, by Markov's inequality, bounded above by

$$
\frac{1}{\delta n^{2}} \sum_{m=k+1}^{N} \mathbb{E}\left[\tau_{m-1}^{m}\right] \leq \frac{2}{\delta n^{2}} \sum_{m=k+1}^{N} t_{m, n} \leq \frac{32 K^{2} C_{0}^{2}}{\delta} \sum_{m=k+1}^{N} \frac{1}{m^{2}} \leq \frac{32 K^{2} C_{0}^{2}}{\delta k},
$$

where $N=\#([-K n, K n] \cap \mathbb{Z})$. This completes the proof of the proposition.

\subsection{Proof of upper bound. We now prove (4.3).}

Single tube case. We first prove (4.3) in the case where $m=1$, which is slightly simpler to explain. Set $T=T_{1}$, and assume that $\partial_{0} T=[a, b] \times\{s\}$. For $\delta>0$, let $\left[T_{\delta}\right]=[T] \cap(\mathbb{R} \times[s+\delta, t]), \partial_{0} T_{\delta}=[a, b] \times\{s+\delta\}$ and $\partial_{1} T_{\delta}=\partial_{1} T$. Clearly, $T_{\delta} \in \mathcal{T}_{\square}$ for small enough $\delta$ and $T_{\delta} \leq T$. Choose $K>0$ large enough that $[T] \subseteq[-K / 2, K / 2] \times \mathbb{R}$. For any $a \in \mathbb{R}$, set $\bar{a}_{\eta}=\left\lfloor a \eta^{-2}\right\rfloor \eta^{2}$.

Since we only care about crossing of the tube $T$, we can work with coalescing random walks on $\mathbb{L}_{\eta}$ that start from every point in $I_{\eta}=[-K, K] \cap \sigma^{-1} \eta \mathbb{Z}$ at time $\bar{s}_{\eta}$ and are killed upon leaving $[-K, K]$. Let $U_{\eta}$ be the number of distinct particles 
in the system at time ${\overline{(s+\delta)_{\eta}}}_{\eta}$, and let $z_{1}^{\eta}, z_{2}^{\eta}, \ldots, z_{U_{\eta}}^{\eta}$ denote the space-time positions on $\mathbb{L}_{\eta}$ of these particles at time $\overline{(s+\delta)}_{\eta}$, enumerated in some predetermined order. Let $\Gamma^{\eta}\left(z_{1}^{\eta}, \ldots, z_{U_{\eta}}^{\eta}\right)$ denote the system of coalescing random walks started from these space-time positions. Observe that if $T$ is crossed by the system of coalescing random walks then necessarily $T_{\delta}$ is crossed by $\Gamma^{\eta}\left(z_{1}^{\eta}, \ldots, z_{U_{\eta}}^{\eta}\right)$.

Now, for all $\varepsilon>0$, by Proposition 4.4 , we can find $k$ so that $\mathbb{P}\left(U_{\eta}>k\right) \leq \varepsilon$. Set $\ell=\limsup _{\eta \rightarrow 0} \mathbf{P}^{\eta}\left(\boxminus_{T}\right)$, and assume that this limsup is achieved along a particular subsequence which we will still denote by $\eta$ with a small abuse of notation. Then by compactness of $[-K, K] \times[s, t]$, we can find a further subsequence (also denoted by $\eta)$ such that $U_{\eta} \rightarrow U$ and $\left(z_{1}^{\eta}, z_{2}^{\eta}, \ldots, z_{k \wedge U_{\eta}}^{\eta}\right) \rightarrow\left(z_{1}, \ldots, z_{k \wedge U}\right)$ in distribution. Along this particular subsequence,

$$
\begin{aligned}
\mathbf{P}^{\eta}\left(\boxminus_{T}\right) & \leq \mathbb{P}\left(T_{\delta} \text { is crossed by } \Gamma^{\eta}\left(z_{1}^{\eta}, \ldots, z_{U_{\eta}}^{\eta}\right)\right) \\
& \leq \varepsilon+\mathbb{P}\left(T_{\delta} \text { is crossed by } \Gamma^{\eta}\left(z_{1}^{\eta}, \ldots, z_{k \wedge U_{\eta}}^{\eta}\right)\right) \\
& \rightarrow \varepsilon+\mathbb{P}\left(T_{\delta} \text { is crossed by } \Gamma\left(z_{1}, \ldots, z_{k \wedge U}\right)\right)
\end{aligned}
$$

by Lemma 4.3, the Markov property and the bounded convergence theorem where $\Gamma\left(z_{1}, \ldots, z_{k \wedge U}\right)$ denotes coalescing Brownian motions started from $\left(z_{1}, \ldots\right.$, $\left.z_{k \wedge U}\right)$. By Theorem 3.1, we conclude

$$
\ell \leq \varepsilon+\mathbf{P}_{\infty}\left(\boxminus_{T_{\delta}}\right)
$$

Since $\varepsilon$ is arbitrary, $\ell \leq \mathbf{P}_{\infty}\left(\boxminus_{T_{\delta}}\right)$. Now, as $\delta \rightarrow 0$, the events $\boxminus_{T_{\delta}}$ are decreasing, so

$$
\lim _{\delta \rightarrow 0} \mathbf{P}_{\infty}\left(\boxminus_{T_{\delta}}\right)=\mathbf{P}_{\infty}\left(\bigcap_{\delta>0} \boxminus_{T_{\delta}}\right) .
$$

Since $\mathscr{H}$ consists of closed collection of tubes, $\bigcap_{\delta>0} \boxminus_{T_{\delta}}=\boxminus_{T}$. Thus,

$$
\ell \leq \mathbf{P}_{\infty}\left(\boxminus_{T}\right)
$$

and so (4.3) holds in the case $m=1$.

Multi tube case. Now assume that $m \geq 1$, and to keep notation simple we will assume that $m=2$. Let $T, T^{\prime}$ be two tubes in $\mathcal{T}_{\square}$ with the start and the end times $s, t$ and $s^{\prime}, t^{\prime}$, respectively. We assume without loss of generality that $s<s^{\prime}$. Reasoning as in the case $m=1$, it is easy to deal with the case where $[T] \cap\left[T^{\prime}\right]=\varnothing$. We thus assume that $[T] \cap\left[T^{\prime}\right] \neq \varnothing$, and hence $s^{\prime}<t$. For $\delta>0$ small enough, let $T_{\delta}$ and $T_{\delta}^{\prime}$ be tubes in $\mathcal{T}_{\square}$ obtained similarly from $T$ and $T^{\prime}$ as in the single tube case. Further, the tube $T_{\delta}$ is decomposed into two tubes $T_{\delta}^{-}$and $T_{\delta}^{+}$in $\hat{\mathcal{T}}$ such that

$$
\left[T_{\delta}^{-}\right]=[T] \cap\left(\mathbb{R} \times\left[s+\delta, s^{\prime}+\delta\right]\right) \quad \text { and } \quad\left[T_{\delta}^{+}\right]=[T] \cap\left(\mathbb{R} \times\left[s^{\prime}+\delta, t\right]\right) .
$$

Choose $K>0$ large enough that $[T],\left[T^{\prime}\right] \subseteq[-K / 2, K / 2] \times \mathbb{R}$. Essentially, we wish to consider coalescing random walks in $\mathbb{L}_{\eta}$ that start from every point in 
$I_{\eta}=[-K, K] \cap \eta \mathbb{Z}$ at time $\bar{s}_{\eta}$ and $\bar{s}_{\eta}^{\prime}$, that are killed upon leaving $[-K, K]$. It is useful to picture the particles starting at time $\bar{s}_{\eta}$ as being colored blue and those starting at time $\bar{s}_{\eta}^{\prime}$ as being colored red. We wish to apply the reasoning of the case $m=1$ separately to all three tubes above, but we need to be a little careful to avoid interactions between the blue and red particles during the interval $\left[s^{\prime}, s^{\prime}+\delta\right]$.

In order to do so, we introduce a coupling of red and blue particles which dominates the coalescing random walks. Here is the precise definition. We start by associating to each vertex $z \in \mathbb{L}_{\eta}$ a random variable $\xi_{z}$ which is an i.i.d. copy of the step distribution $\xi$. Coalescing random walk $\left(S_{k}, t_{k}\right)_{k \geq 0}$ in $\mathbb{L}_{\eta}$ from space-time point $z=\left(x_{0}, t_{0}\right) \in \mathbb{L}_{\eta}$ may be defined by setting, $S_{0}=x_{0}$ and for $k \geq 0$,

$$
S_{k+1}=S_{k}+\sigma^{-1} \eta \xi_{\left(S_{k-1}, t_{k-1}\right)} \quad \text { and } \quad t_{k}=t_{0}+k \eta^{2} .
$$

In order to prevent the blue and red particles from interacting during the interval $\left[{\overline{s^{\prime}}}_{\eta}, \overline{\left(s^{\prime}+\delta\right)_{\eta}}\right]$, we modify this description as follows. Consider all the points $z \in$ $I_{\eta} \times\left[{\overline{s^{\prime}}}_{\eta},{\overline{\left(s^{\prime}+\delta\right)_{\eta}}}_{\eta} \cap \mathbb{L}_{\eta}\right.$. We endow each such $z$ with a new independent copy $\xi_{z}^{\prime}$ of $\xi$ in addition to the original $\xi_{z}$. Then a blue particle will use the random variable $\xi_{z}$ to move forward from space-time point $z$, but a red particle will always use the random variable $\xi_{z}^{\prime}$ if it has the choice between $\xi_{z}$ and $\xi_{z}^{\prime}$ (otherwise it uses $\xi_{z}$ ). The particles still get killed upon exiting the interval $[-K, K]$. Note that as a result of this definition, after time ${\overline{\left(s^{\prime}+\delta\right)}}_{\eta}$, if a red and a blue particle are on the same site then they coalesce and necessarily follow the same path afterward. We may thus think of the resulting particle as carrying both the red and blue colours (see Figure 4).

Then observe that if $\boxminus_{T} \cap \boxminus_{T^{\prime}}$ occurs then necessarily the following three things must occur:

(a) $T_{\delta}^{-}$is crossed by the blue particles.

(b) $T_{\delta}^{+}$is crossed by the remaining blue particles at time ${\overline{\left(s^{\prime}+\delta\right)}}_{\eta}$.

(c) $T_{\delta}^{\prime}$ is crossed by the remaining (blue or red) particles at time $\overline{\left(s^{\prime}+\delta\right)_{\eta}}$.

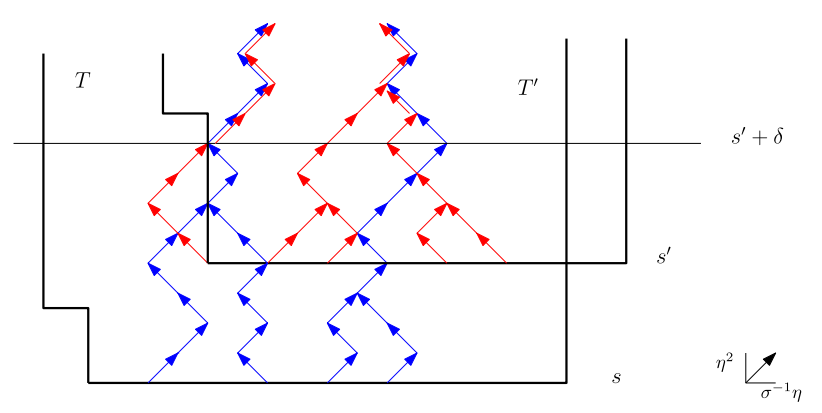

FIG. 4. The coupling of partially coalescing (simple) random walks. 
Let $U_{\eta}^{-}$be the number of distinct blue particles in the system at time $\overline{(s+\delta)}$, and let $z_{1}^{\eta}, z_{2}^{\eta}, \ldots, z_{U_{\eta}^{-}}^{\eta}$ denote the space-time positions on $\mathbb{L}_{\eta}$ of these particles. Let $\Gamma_{-}^{\eta}=\Gamma_{-}^{\eta}\left(z_{1}^{\eta}, \ldots, z_{U_{\eta}^{-}}^{\eta}\right)$ denote the system of coalescing random walks started from these space-time positions, and ended at time $\overline{\left(s^{\prime}+\delta\right)_{\eta}}$. Let $U_{\eta}^{+}$ denote the number of blue particles in $\Gamma_{-}^{\eta}\left(z_{1}^{\eta}, \ldots, z_{U_{\eta}-}^{\eta}\right)$ left at time $\overline{\left(s^{\prime}+\delta\right)_{\eta}}$. Let also $U_{\eta}^{\prime}$ denote the number of distinct red particles at time $\overline{\left(s^{\prime}+\delta\right)_{\eta}}$. Denote by $w_{1}^{\eta}, \ldots, w_{U_{\eta}^{+}}^{\eta}, y_{1}^{\eta}, \ldots, y_{U_{\eta}^{\prime}}^{\eta}$ the locations of the blue and red particles at time $\overline{\left(s^{\prime}+\delta\right)_{\eta}}$, and denote by $\Gamma_{+}^{\eta}=\Gamma_{+}^{\eta}\left(w_{1}^{\eta}, \ldots, w_{U_{\eta}^{+}}^{\eta}, y_{1}^{\eta}, \ldots, y_{U_{\eta}^{\prime}}^{\eta}\right)$ the collection of coalescing blue and red particles started from these positions at time $\overline{\left(s^{\prime}+\delta\right)_{\eta}}$. Note that blue and red particles are allowed to coalesce after this time (in which case they count as both blue and red particles for what follows).

From the above discussion, it follows that

$$
\mathbf{P}^{\eta}\left(\boxminus_{T} \cap \boxminus_{T^{\prime}}\right) \leq \mathbb{P}\left(T_{\delta}^{-}, T_{\delta}^{+} \text {are crossed by } \Gamma_{-}^{\eta} \text { and } T_{\delta}^{\prime} \text { is crossed by } \Gamma_{+}^{\eta}\right) .
$$

As in the case $m=1$ set $\ell=\lim \sup _{\eta \rightarrow 0} \mathbf{P}^{\eta}\left(\boxminus_{T} \cap \boxminus_{T^{\prime}}\right)$, and assume that this limsup is achieved along a particular subsequence which we will still denote $\eta$ with a small abuse of notation. For all $\varepsilon>0$, by Proposition 4.4, we can find $k$ so that $\mathbb{P}\left(\max \left(U_{\eta}^{-}, U_{\eta}^{\prime}\right)>k\right) \leq \varepsilon$ for all $\eta$. Then by compactness, we can find a further subsequence (also denoted by $\eta$ ) such that $\left(U_{\eta}^{-}, U_{\eta}^{\prime}\right) \rightarrow\left(U^{-}, U^{\prime}\right)$ and the two vectors $\left(z_{1}^{\eta}, \ldots, z_{k \wedge U_{\eta}^{-}}^{\eta}\right)$ and $\left(y_{1}^{\eta}, \ldots, y_{U_{\eta}^{\prime} \wedge k}^{\eta}\right)$ converge jointly to two vectors $\left(z_{1}, \ldots, z_{k \wedge U^{-}}\right)$and $\left(y_{1}, \ldots, y_{k \wedge U^{\prime}}\right)$ in distribution. Let $\Gamma(z, y)$ denote coalescing Brownian motions starting from $\left(z_{1}, \ldots, z_{k \wedge U^{-}}, y_{1}, \ldots, y_{k \wedge U^{+}}\right)$. Applying the Markov property in (4.7) repetitively and by bounded convergence theorem, we see after taking the limit that along this particular subsequence:

$$
\begin{aligned}
\ell & \leq \varepsilon+\mathbb{P}\left(T_{\delta}, T_{\delta}^{\prime} \text { are crossed by } \Gamma(z, y)\right) \\
& \leq \varepsilon+\mathbf{P}_{\infty}\left(\boxminus_{T_{\delta}} \cap \boxminus_{T_{\delta}^{\prime}}\right) .
\end{aligned}
$$

From there, we conclude as in (4.5), since $\varepsilon>0$ is arbitrary and $\mathscr{H}$ consists of closed collections of tubes,

$$
\ell \leq \mathbf{P}_{\infty}\left(\boxminus_{T} \cap \boxminus_{T^{\prime}}\right) .
$$

This proves (4.3), and hence Theorem 4.1.

\section{Coalescing flow on Sierpinski gasket.}

5.1. Sierpinski gasket. Let $H_{0}$ be the unit triangle in $\mathbb{R}^{2}$ with vertices $\{(0,0),(1,0),(1 / 2, \sqrt{3} / 2)\}$. The finite Sierpinski gasket is a fractal subset of the plane that can be constructed via the following Cantor-like cut-out procedure. Let $\left\{b_{0}, b_{1}, b_{2}\right\}$ be the midpoints of three sides of $H_{0}$ and let $A$ be the interior of the 
triangle with vertices $\left\{b_{0}, b_{1}, b_{2}\right\}$. Define $H_{1}:=H_{0} \backslash A$ so that $H_{1}$ is the union of 3 closed upward facing triangles of side length $2^{-1}$. Now repeat this operation on each of the smaller triangles to obtain a set $\mathrm{H}_{2}$, consisting of 9 upward facing closed triangles, each of side $2^{-2}$. Repeating this procedure, we have a decreasing sequence of closed nonempty sets $\left\{H_{n}\right\}_{n=0}^{\infty}$ and we define the finite Sierpinski gasket as

$$
G_{\text {fin }}:=\bigcap_{n=0}^{\infty} H_{n} .
$$

We call the unbounded set

$$
G:=\bigcup_{n=0}^{\infty} 2^{n} G_{\text {fin }}
$$

the infinite Sierpinski gasket. We equip it with the shortest path metric $\rho_{G}$ which is comparable to the usual Euclidean metric $|\cdot|$ (see, e.g., [5], Lemma 2.12) with the relation,

$$
|x-y| \leq \rho_{G}(x, y) \leq c|x-y| \quad \forall x, y \in G,
$$

for a suitable constant $1<c<\infty$. Let $\mu$ denote the $d_{f}$-dimensional Hausdorff measure on $G$ where $d_{f}:=\log 3 / \log 2$ is the fractal or mass dimension of the gasket. The following estimate on the volume growth of $\mu$ is known (see [6]):

$$
\mu(B(x, r)) \leq C r^{d_{f}} \quad \text { for } x \in G, 0<r<1,
$$

where $B(x, r) \subseteq G$ is the open ball with center $x$ and radius $r$ in the Euclidean metric.

For each $n \in \mathbb{Z}$, the set $2^{m} H_{m+n}$ is made up of $3^{m+n}$ triangles of side length $2^{-n}$ whenever $m \geq n$. Each of those triangles are called an $n$-triangle of $G$. Denote by $\mathcal{S}_{n}$ the collection of all $n$-triangles of $G$. Let $\mathcal{V}_{n}$ be the set of vertices of the $n$-triangles. We will restrict our attention to infinite Sierpinki gasket while constructing the coalescing Brownian flow. The case of finite gasket can also be dealt with very similar arguments.

5.2. Brownian motion on gasket. We construct a graph $G_{n}$ embedded in the plane with vertices $\mathcal{V}_{n}$ by adding edges between pairs of vertices that are distance $2^{-n}$ apart from each other. Let $X^{n}$ be the nearest-neighbor random walk on $G_{n}$ simultaneously defined on the same probability space. It is known (see $[5,6])$ that the sequence $\left(X_{\left\lfloor 5^{n} t\right\rfloor}^{n}\right)_{t \geq 0}$ converges almost surely as $n \rightarrow \infty$ to a limiting process $\left(X_{t}\right)_{t \geq 0}$ that is a $G$-valued strong Markov process (indeed, a Feller process) with continuous sample paths. The process $X$ is naturally called the Brownian motion on the gasket. It has the following scaling property:

$$
\left(2 X_{t}\right)_{t \geq 0} \text { under } \mathbb{P}^{x} \text { has same law as }\left(X_{5 t}\right)_{t \geq 0} \text { under } \mathbb{P}^{2 x} \text {. }
$$


The process $X$ has a symmetric transition density $p_{t}(x, y), x, y \in G, t>0$ with respect to the measure $\mu$ that is jointly continuous on $(0, \infty) \times G \times G$. Let $d_{w}:=$ $\log 5 / \log 2$ denote the walk dimension of the gasket. The following crucial "heat kernel bound" is established in [6]

$$
p_{t}(x, y) \leq c_{1} t^{-d_{f} / d_{w}} \exp \left(-c_{2}\left(\frac{|x-y|^{d_{w}}}{t}\right)^{1 /\left(d_{w}-1\right)}\right) .
$$

A matching lower bound (with different constants $c_{3}$ and $c_{4}$ ) also exists. This shows that the Brownian motion on the gasket is sub-diffusive.

5.3. Coalescing Brownian flow on gasket. We now state the analogue of Theorem 3.1 in the case of the Sierpinski gasket. Let $\mathcal{D}=\left\{z_{1}, z_{2}, \ldots\right\}$ be a countable ordered set which is dense in $G \times \mathbb{R}$ where $z_{i}=\left(x_{i}, t_{i}\right)$. Let $\left(W_{j}\right)_{j \geq 1}$ be an independent family of Brownian motions on the Sierpinski gasket $G$ defined on a common probability space $(\Omega, \mathcal{F}, \mathbb{P})$, started from time $t_{j}$ at position $x_{j}$. We can apply the coalescing rule to obtain a collection $\Gamma_{n}$ of $n$ coalescing Brownian motions on $G$ denoted by $W_{1}^{c}, W_{2}^{c}, \ldots, W_{n}^{c}$. Let us define $\mathcal{W}_{n}$ to be, as before, the set of all tubes (now $d=2$ ) crossed by $\Gamma_{n}$, that is, $\mathcal{W}_{n}=\mathcal{W}\left(z_{1}, \ldots, z_{n}\right):=\operatorname{Cr}\left(\Gamma_{n}\right) \in \mathscr{H}$. The next theorem defines the coalescing Brownian flow on the gasket and its proof is a straightforward adaptation of the arguments in the proof of Theorem 3.1 (we leave the details to the reader).

THEOREM 5.1. As $n \rightarrow \infty, \mathcal{W}_{n}$ converges in distribution to a random variable $\mathcal{W}_{\infty}$, whose law does not depend on $\mathcal{D}$ (including its order).

Definition 5.2. A random variable on $\mathscr{H}$ with law $\mathbf{P}_{\infty}$ is called a coalescing Brownian flow on the Sierpinski gasket.

5.4. Characterization. In a way which is analogous to Theorem 3.4 , we state a useful characterization of the coalescing Brownian flow on the Sierpinski gasket. The result will be formally very similar to Theorem 3.4 but we will work with a slightly different class of tubes, chosen so that they are more suited to the geometry of the Sierpinski gasket. Let $\triangle_{0}$ denote the convex hull of the vertices 0,1 and $e^{i \pi / 3}$ in $\mathbb{R}^{2}$. Let $\mathbb{T}_{n}$ be the triangular lattice on the plane with mesh size $2^{-n}$ (so that $G_{n}$ is a subgraph of $\mathbb{T}_{n}$ ). Define

$$
\mathcal{E}=\left\{z+\frac{1}{2^{n}} \triangle_{0}: n \in \mathbb{Z}, z \in \mathbb{T}_{n}\right\} .
$$

Note that if $\triangle \in \mathcal{E}, \triangle$ is an upward-facing equilateral triangle in $\mathbb{T}_{n}$ for large enough $n$. The Brownian motion starting at some point inside $\triangle$ can escape $\triangle$ only through one of the three vertices of $\triangle$.

DEFinition 5.3 (A dense family of triangular tubes). Let $\mathcal{T}_{\triangle}$ be the family of all tubes $T$ such that: 
(a) The set [T] can be expressed as an union of a finite number of cylinders (triangular prisms) of the form $\triangle \times[s, t]$ with $s<t$, for some $\triangle \in \mathcal{E}$.

(b) $\partial_{0} T=[T] \cap\left(\mathbb{R}^{2} \times\left\{t_{0}\right\}\right)$ and $\partial_{1} T=[T] \cap\left(\mathbb{R}^{2} \times\left\{t_{1}\right\}\right)$, where $t_{0}$ and $t_{1}$ are the start and the end time of $T$.

One can check that $\mathcal{T}_{\triangle}$ is dense in $\mathcal{T}$ because every downward-facing triangle is a subset of an upward-facing triangle of twice its size.

We now state our characterization.

THEOREM 5.4. $\quad \mathbf{P}_{\infty}$ is the unique probability measure on $\mathscr{H}$ such that for all $m \geq 1$ and for all fixed tubes $T_{1}, \ldots, T_{m} \in \mathcal{T}_{\triangle}$,

$$
\begin{aligned}
& \mathbf{P}_{\infty}\left(\boxminus_{T_{1}} \cap \cdots \cap \boxminus_{T_{m}}\right) \\
& \quad=\sup _{n \geq 1 ; z_{1}, \ldots, z_{n} \in G \times \mathbb{R}} \mathbb{P}\left(\mathcal{W}\left(z_{1}, \ldots, z_{n}\right) \in \boxminus_{T_{1}} \cap \cdots \cap \boxminus_{T_{m}}\right) .
\end{aligned}
$$

PROOF. The proof of Theorem 3.4 can be adapted as follows. Lemma 3.5, which relies on the fact that Arratia's flow comes down from infinity, will now use Theorem 5.1 in [11] which says that the same is true even for coalescing Brownian motion on the gasket. But Lemma 3.6 needs a different argument, since it relies on scale invariance of (real) Brownian motion at all scales. Since Brownian motion on the gasket is scale-invariant only for a discrete set of scales (which does not come arbitrarily close to 1 ), this lemma needs a different proof.

Thus, let $T \in \mathcal{T}_{\triangle}$ be fixed and let $\partial_{0} T=B \times\left\{t_{0}\right\}$, where $B$ is the union of finitely many triangles from $\mathcal{E}$. To keep the presentation simpler we will assume that $B=\triangle$ for some fixed equilateral triangle $\triangle$ in $\mathcal{E}$ of side 1 . Let $t_{e}>0$ be such that $T \cap\left(\mathbb{R}^{2} \times\left[t_{0}, t_{0}+t_{e}\right]\right)=\Delta \times\left[t_{0}, t_{0}+t_{e}\right]$. Let $t_{1}=t_{0}+\delta$ and $t_{2}=t_{1}+\alpha$, where $\alpha=t_{e} / 4$. Fix a countable dense set $\mathcal{D}$ of $G \times \mathbb{R}$ and consider a countable system of coalescing Brownian particles starting from the space-time points in $\mathcal{D}$. Let $A_{\triangle}(s, t)$ denote the set of locations at time $t$ of the particles that started at some time before $s$ and were in $\triangle$ throughout the time interval $[s, t]$. For $\delta>0$, let $\triangle^{\delta}$ be the $\delta$-enlargement of $\triangle$. We wish to show that for all $\varepsilon>0$, we can choose $\delta>0$ so that $A_{\triangle}\left(t_{1}, t_{2}\right)=A_{\triangle \delta}\left(t_{0}, t_{2}\right)$ with probability greater than $1-\varepsilon$. Again, by continuity of the distribution of $\left|A_{\triangle}(s, t)\right|$ in $s$ and $t$, it suffices to show the following.

LEMMA 5.5. Given $\varepsilon>0$, there exists $\delta_{0}>0$ such that for all $\delta \leq \delta_{0}$, the event $\left|A_{\triangle}\left(t_{1}, t_{2}\right)\right|=\left|A_{\triangle}\left(t_{1}, t_{2}\right)\right|$ holds with probability greater than $1-\varepsilon$.

PROOF. Let $v_{1}, v_{2}, v_{3}$ be the vertices of the triangle $\triangle$. Call the vertex $v_{i}$ an exit point of $\triangle$ if the set $B\left(v_{i}, \lambda\right) \backslash \triangle$ has nonempty intersection with $G$ for each $\lambda>0$. Similarly, call the vertex $v_{i}$ an entry point of $\triangle$ if the set $B\left(v_{i}, \lambda\right) \backslash\left\{v_{i}\right\}$ has nonempty intersection with $\Delta \cap G$ for each $\lambda>0$. For the vertex $v_{i}$, let $q_{k}^{i}$ (resp., $r_{k}^{i}$ ) be the union of one or two (resp., one) $k$-triangle(s) in $\mathcal{S}_{k}$ attached to $v_{i}$ and 


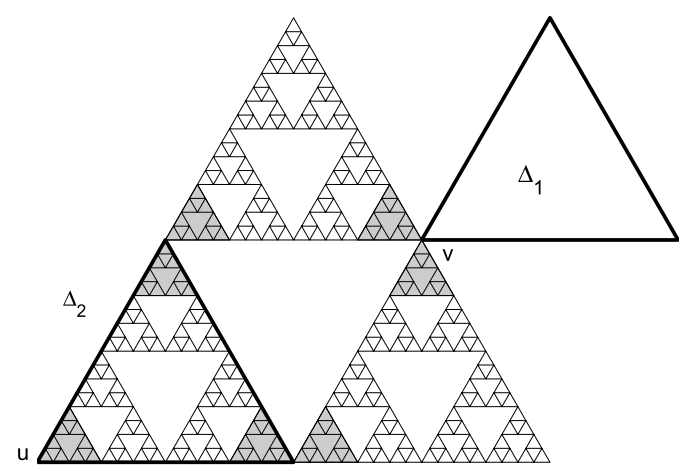

FIG. 5. The triangles $\Delta_{1}, \triangle_{2} \in \mathcal{E}$ are shown with thick lines. For example, $u$ is an entry point of $\Delta_{1}$ but not an exit point where as $v$ is an exit point of $\Delta_{2}$ but not an entry point. The shaded region attached to $v$ is $q_{k}^{v}$ and the shaded triangle attached to $u$ is $r_{k}^{u}($ for $k=3)$.

lying outside (resp., inside) of $\triangle$, if $v_{i}$ is an exit (resp., entry) point of $\triangle$ and the empty set otherwise (see Figure 5). Since $\Delta$ is an upward-facing triangle adapted to the triangular lattice $\mathbb{T}_{0}$, the difference between $\triangle^{\delta} \cap G$ and $\triangle \cap G$ is "tiny" when $\delta$ is small. Indeed, for $\delta=2^{-(k+1)},\left(\triangle^{\delta} \backslash \triangle\right) \cap G \subseteq q_{k}^{1} \cup q_{k}^{2} \cup q_{k}^{3}$. Thus, we can assume that each $v_{i}$ is an exit point of $\triangle$, if not we can safely ignore it for the rest of the proof. For $\delta=2^{-(k+1)}$, let $\Delta^{-\delta}$ be the closed set obtained by removing $r_{k}^{1}, r_{k}^{2}$ and $r_{k}^{3}$ from $\triangle$. We first show that:

CLAIM 1. One can choose $\delta_{1}>0$ small enough that with probability at least $1-\varepsilon / 2$, no particle starting in $\Delta^{\delta} \backslash \Delta^{-\delta}$ at time $t_{1}$ can stay inside $\Delta^{\delta}$ up to time $t_{2}$, for any $\delta<\delta_{1}$.

The proof of the above claim relies on the following further claim.

Claim 2. Given $\varepsilon_{1}>0$ and $\gamma>0$, there exists $k_{0}$, such that for all $k \geq k_{0}$, the total coalescence time of the countable particles starting from any $k$-triangle of $G$ is less than $\gamma$ with probability $1-\varepsilon_{1}$.

Proof of Claim 1. To see this, note that for $\delta=2^{-(k+1)}$, the set $\Delta^{\delta} \backslash \triangle^{-\delta}$ consists of three connected regions, say $\ell_{k}^{1}, \ell_{k}^{2}$ and $\ell_{k}^{3}$, of diameter bounded by $2^{-(k-1)}$ where $\ell_{k}^{i}=q_{k}^{i} \cup r_{i}^{k}$. Consider the particles starting from $\ell_{k}^{i}, 1 \leq i \leq 3$ at time $t_{1}$. By Claim 2, for $\gamma>0$ fixed but sufficiently small, at time $t_{1}+\gamma$, the number of descendants of these particles is one with probability at least $1-\varepsilon / 4$, and hence coincides with the particle starting from $v_{i}$ at time $t_{1}$. By choosing $\gamma>0$ small and $\delta>0$ even smaller, the particle starting from $v_{i}$ at time $t_{1}$ is guaranteed to exit the triangle $q_{k}^{i}$ via one of its vertices other than $v_{i}$ between time $t_{1}+\gamma$ and $t_{2}$ with probability $1-\varepsilon / 4$. On the intersection of these two events (which has probability at least $1-\varepsilon / 2$ ), no particle starting from $\ell_{k}^{i}$ can cross $\triangle^{\delta} \times\left[t_{1}, t_{2}\right]$. 
Proof of Claim 2. We first claim that there exist $n \geq 1$ and $u \in(0,1)$, depending only on $\varepsilon_{1}$, such that with probability at least $1-\varepsilon_{1} / 2$, the number of descendants at time $u$ of the particles starting from any 0-triangle $L$ in $\mathcal{S}_{0}$ is less than $n$ and moreover, those particles are inside $L+B(0,1 / 2)$ at time $u$. The main content of the claim lies in the fact that $n$ and $u$ can be chosen independent of $L$. This follows from Lemma 5.6 of [11], which actually gives much more in terms of quantitative bounds. Thus, by scaling, for any $k \geq 1$, with probability at least $1-\varepsilon_{1} / 2$, the number of descendants at time $u 5^{-k}$ of particles starting from any $k$-triangle $L$ is less than $n$, and moreover, those particles are inside $L+$ $B\left(0,2^{-(k+1)}\right)$ at time $u 5^{-k}$. Now choose $k_{0}$ large enough such that for all $k \geq$ $k_{0}$ two independent Brownian motions starting within distance $2^{-(k-1)}$ from one another at time 0 will meet by time $\gamma / 2$ with probability at least $1-\varepsilon_{1} /(2 n)$. This is possible by (6.6) whose proof is given in Section 6.2. This completes the proof of Claim 2.

Now we will continue with the proof of Lemma 5.5. Consider the particles starting from $\triangle^{-\delta_{1}}$ at time $t_{1}$. We have to argue that for sufficiently small $\delta$ with probability $1-\varepsilon / 2$, no such particle can stay within $\triangle^{\delta}$ between time $\left[t_{1}, t_{2}\right]$ but go outside $\triangle$ at some time in $\left[t_{1}, t_{2}\right]$. Let $\Xi_{t}$ be the closure of the locations of the coalescing particles at time $t_{1}+t$ which were in the compact set $\triangle^{-\delta_{1}}$ at time $t_{1}$. It was proved in [11], Theorem 5.2, that $\Xi_{t} \rightarrow \Xi_{0}=\Delta^{-\delta_{1}}$ in probability as $t \rightarrow 0+$ in the Hausdorff metric. Consequently, we can find $\eta>0$ sufficiently small such that no particle which was inside $\Delta^{-\delta_{1}}$ at time $t_{1}$ can leave $\triangle$ before time $t_{1}+\eta$, with probability greater than $1-\varepsilon / 6$. By coming down from infinity, choose $n$ sufficiently large, so that $\left|A_{\triangle}\left(t_{1}, t_{1}+\eta\right)\right| \leq n$ with probability at least $1-\varepsilon / 6$.

Now the lemma will follow if we show that for any particle, say $X$, which is inside $\triangle$ at time $t_{1}+\eta$, the probability that it leaves $\Delta$ some time between $t_{1}+\eta$ and $t_{2}$ but always stays inside $\triangle^{\delta}$ within time $\left[t_{1}+\eta, t_{2}\right]$ is at most $\varepsilon_{0}=\varepsilon /(6 n)$. Let $\tau$ be time when $X$ hits one of the vertices of $\Delta$ for the first time after $t_{1}+\eta$. We find $\beta \in\left(0, t_{2}-t_{1}-\eta\right)$ small such that $\mathbb{P}\left(\tau \in\left[t_{2}-\beta, t_{2}\right]\right) \leq \varepsilon_{0} / 2$, uniformly over the position of $X$ in $\triangle$ at time $t_{1}+\eta$. If $\tau \leq t_{2}-\beta$, then $X_{\tau}$ will be at one of the vertices $v_{i}$ at time $\tau$ and by choosing $\delta$ small, it will leave $\triangle^{\delta}$ some time during the time interval $[\tau, \tau+\beta]$ with probability at least $1-\varepsilon_{0} / 2$.

Putting together these observations we have completed the proof of the lemma.

The rest of the proof of Theorem 5.4 is a straightforward adaptation of Lemmas 3.7 and 3.8, which is left to the reader. Note in particular that for a tube $T \in \mathcal{T}_{\triangle}$ with $[T]=\bigcup_{j=1}^{k} \triangle_{j} \times\left[s_{j}, t_{j}\right]$, the set of corner points of $T$ is a subset of $\left\{\left(v_{i j}, s_{j}\right),\left(v_{i j}, t_{j}\right): 1 \leq i \leq 3,1 \leq j \leq k\right\}$, and hence is finite. Here, $v_{1 j}, v_{2 j}$ and $v_{3 j}$ denote the vertices of the triangle $\triangle_{j} \in \mathcal{E}$. 
6. Invariance principle for coalescing random walks on gasket. Let $\eta=$ $2^{-n}$ and consider an infinite Sierpinsky gasket $G_{n}$ with mesh size $2^{-n}$. In this section, we consider coalescing random walks on $G_{n}$ defined as follows: initially there is a particle at every vertex of $G_{n}$. They perform independent simple random walks, jumping every $5^{-n}$ units of time, and coalesce when they are on the same vertex of $G_{n}$. Consider the law $\mathbf{P}^{\eta}$ on $\mathscr{H}$ that these particles induce.

THEOREM 6.1. As $\eta \rightarrow 0$,

$$
\mathbf{P}^{\eta} \rightarrow \mathbf{P}_{\infty}
$$

weakly, where $\mathbf{P}_{\infty}$ is the law of the coalescing Brownian flow on $G$, as defined in Theorem 5.1.

PROOF. The proof of Theorem 6.1 follows the same outline as in the Brownian case. However, two important facts are needed to make the strategy applicable. The first is a statement about "uniform coming down from infinity" (stated below in Proposition 6.2). The second is an intuitively obvious statement that finitely many coalescing random walks converge to the same number of coalescing Brownian motions. This will be stated in Proposition 6.6. This is probably well known in the folklore, but we could not find a reference for it.

6.1. Uniform coming down from infinity on the gasket. Consider the setup above, with coalescing random walks started at time 0 on $G_{n}$, and jumping every $5^{-n}$ units of time. Give a bounded region $T \subset \mathbb{R}^{2}$, suppose that the random walk particles are killed as soon as they touch $\mathbb{R}^{2} \backslash T$. Let $N(t)$ denotes the number of particles left at time $t$. The following is the analogue of Proposition 4.4 but for the case of the gasket. As before, this is essentially the only place where one needs some quantitative estimates about coalescing random walks.

Proposition 6.2 (Uniform coming down from infinity). For every $\delta>0$ and $\varepsilon>0$, there exists $k \geq 0$ depending only on $T$, such that $\mathbb{P}(N(\delta)>k) \leq \varepsilon$ for all sufficiently large $n$.

PROOF. The proof depends on two simple lemmas. In the rest of the proof, we assume without loss of generality that $T$ is the unit equilateral triangle with apices at $z=0, z=1$, and $z=e^{i \pi / 3}$ when viewed as a subset of the complex plane.

LEMMA 6.3. There exists a universal constant $0<C<\infty$ such that the following holds for all $1 \leq k \leq n$. Let $X, Y$ be two independent (unkilled) random walks started at $x, y \in \bar{G}_{n}$ such that $|x-y| \leq 2^{-k}$. Then

$$
\mathbb{P}\left(\tau<5^{-k}\right) \geq \frac{1}{C}
$$

uniformly on $x, y \in G_{n}$ such that $|x-y| \leq 2^{-k}$, where $\tau$ is the first meeting time of $X$ and $Y$. 
PROOF. This relies on uniform heat-kernel estimates of the random walks on the gasket due to Jones [15]. To use this, it is convenient to use a different scaling of space and time: thus consider $G_{0}=2^{n} G_{n}$ and let the random walks $X, Y$ make jumps at integer times. Then Lemma 6.3 is equivalent to the statement that, uniformly over $x, y \in G_{0}$ with $|x-y| \leq 2^{k}, \mathbb{P}\left(\tau<5^{k}\right) \geq 1 / C$. Let $p_{t}(x, y)=\mathbb{P}\left(X_{t}=y \mid X_{0}=x\right)$ be the transition density function of the random walk $X$. Now, Theorem 18 of [15] states that for all $t \geq c_{0}|x-y| \vee t_{0}$,

$$
p_{t}(x, y) \geq c_{1} t^{-d_{s} / 2} \exp \left(-c_{2}\left(\frac{|x-y|^{d_{w}}}{t}\right)^{1 /\left(d_{w}-1\right)}\right),
$$

where

$$
d_{s}=\frac{2 \log 3}{\log 5} ; \quad d_{w}=\frac{\log 5}{\log 2} .
$$

Likewise, Theorem 17 of [15] states that for all $t \geq c_{0}|x-y| \vee t_{0}$,

$$
p_{t}(x, y) \leq c_{3} t^{-d_{s} / 2} \exp \left(-c_{4}\left(\frac{|x-y|^{d_{w}}}{t}\right)^{1 /\left(d_{w}-1\right)}\right) .
$$

Let $J$ denote the number of intersections of the walks $X, Y$ during the time interval $\left[1,5^{k}\right]$. Then by reversibility, and (6.1), for $k$ large,

$$
\begin{aligned}
\mathbb{E}[J] & =\sum_{s=1}^{5^{k}} \sum_{z \in G_{0}} \mathbb{P}\left(X_{s}=Y_{s}=z\right)=\sum_{s=1}^{5^{k}} p_{2 s}(x, y) \\
& \geq c_{1} \sum_{s=(1 / 2) 5^{k}}^{5^{k}} \frac{1}{\left(5^{k}\right)^{\log 3 / \log 5}} \exp \left(-c_{2}\left(\frac{\left(2^{k}\right)^{\log 5 / \log 2}}{5^{k}}\right)^{1 /\left(d_{w}-1\right)}\right) \geq c(5 / 3)^{k} .
\end{aligned}
$$

On the other hand, by (6.3), for $k$ large,

$$
\begin{aligned}
\mathbb{E}\left[J^{2}\right] & \leq \mathbb{E}[J]+2 \sum_{1 \leq s<t \leq 5^{k}} \mathbb{P}\left(X_{s}=Y_{s}\right) \sup _{w \in G_{0}} p_{2(t-s)}(w, w) \\
& \leq \mathbb{E}[J]+2 \sum_{s=1}^{5^{k}} \mathbb{P}\left(X_{s}=Y_{s}\right) \sum_{s<t \leq 5^{k}} \frac{c^{\prime}}{(t-s)^{\log 3 / \log 5}} \\
& \leq \mathbb{E}[J]+c^{\prime} \sum_{s=1}^{5^{k}} \mathbb{P}\left(X_{s}=Y_{s}\right)(5 / 3)^{k} \leq c^{\prime}(5 / 3)^{k} \mathbb{E}[J] .
\end{aligned}
$$

Thus, by the Payley-Zygmund inequality,

$$
\mathbb{P}(J>0) \geq \frac{\mathbb{E}[J]^{2}}{\mathbb{E}\left[J^{2}\right]} \geq \frac{\mathbb{E}[J] \cdot c(5 / 3)^{k}}{c^{\prime}(5 / 3)^{k} \mathbb{E}[J]} \geq c / c^{\prime},
$$

as required. 
REMARK 6.4. Note that Lemma 6.3 implicitly relies on the fact that the simple random walk on $G_{n}$ is aperiodic [similarly as the distribution $\xi$ in (4.1)]. Though we do not directly appeal to aperiodicity in our proof, it is used in Jones' lower bound estimate (6.1).

Our second lemma is an induction scheme which is inspired by an argument in [11] for the fact that coalescing Brownian particles come down from infinity. See also [2] where a similar argument is used.

LeMma 6.5. Fix $t>0$ and let $m$ be such that $3^{m+1} \leq N=N(t)<3^{m+2}$. Then there exists an absolute constant $\theta<1$, such that by time $t^{\prime}=t+5^{-m}$, $N\left(t^{\prime}\right) \leq \theta N(t)$ with probability greater than $1-e^{-c N}$.

PROOF. We first claim that it is possible to find a pairing of the particles $\left(X_{i}, Y_{i}\right)_{1 \leq i \leq N / 2}$ such that $\left|X_{i}(t)-Y_{i}(t)\right| \leq 2^{-m}$ for all $1 \leq i \leq \frac{N}{3}$. Indeed, tile $T$ with $3^{m}$ triangles of side length $2^{-m}$. Within each such triangle, pair as many particles as possible. This leaves at most one unpaired particle per triangle, and we pair these arbitrarily.

Having constructed this pairing, consider now a partial coalescing system in which coalescence occurs only between matched particles, and distinct pairs of particles evolve completely independently of one another. By the monotonicity property of coalescing random walks, it is easy to argue (see [11]) that the partial system dominates in distribution the fully coalescing system. Hence, it suffices to prove the claim on the partially coalescing system.

By Lemma 6.3 and by the strong Markov property, at time $t^{\prime}$, each pair $\left(X_{i}, Y_{i}\right)$ with $1 \leq i \leq \frac{N}{3}$ has a probability at least $1 / C$ to have coalesced, and these events are independent of one another. (Note further that the number of particles may decrease further due to particles leaving the region $T$, but this can only help us.) Thus, $N\left(t^{\prime}\right) \leq N-\left(1 / C^{\prime}\right)(N / 3)$ with probability greater than $1-\exp \left(-c N^{\prime}\right)$, by an easy large deviation bound on the binomial random variables. Taking $\theta=$ $1-1 /\left(4 C^{\prime}\right)$ gives the desired result.

With these two lemmas, we can now complete the proof of the proposition.

Let $\tau_{n}=0$, and define for $m \leq n, \tau_{m}=\inf \left\{t \geq 0: N(t) \leq 3^{m}\right\}$. By iterating Lemma $6.5\left\lceil\log _{\theta^{-1}} 3\right\rceil$ times, we see that there exists $C>0$ such that

$$
\mathbb{P}\left(\tau_{m}-\tau_{m-1} \geq C 5^{-m}\right) \leq C \exp \left(-3^{m} / C\right) .
$$

Let $A_{m}$ denote the complement of the event above, and let $A=\bigcap_{m=M}^{n} A_{m}$, where $M$ is a fixed large number. Then on the one hand, by (6.4),

$$
\mathbb{P}\left(A^{c}\right) \leq C \exp \left(-3^{M} / C\right)
$$

uniformly in $n$. On the other hand, on the event $A$,

$$
\tau_{M} \leq C 5^{-M}+\cdots+C 5^{-n} \leq C 5^{-M} .
$$


Thus, if $\delta>0, \varepsilon>0$ are fixed as in the statement of the proposition, we choose $M$ large enough that $C 5^{-M} \leq \delta$ and $C \exp \left(-3^{M} / C\right) \leq \varepsilon$. Then picking $k=3^{M}$, we obtain

$$
\mathbb{P}(N(\delta)>k) \leq \mathbb{P}\left(\tau_{M} \geq \delta\right) \leq \mathbb{P}\left(A^{c}\right) \leq \varepsilon .
$$

Noting that the choice of $M$ (and thus of $k$ ) depends only on $\delta$ and $\varepsilon$ (and not on $n$ ) completes the proof.

6.2. Finitely many coalescing random walks. Fix $n \geq 1$ and let $z_{1}, \ldots, z_{n} \in$ $G \times \mathbb{R}$. For $\eta=2^{-m}$, let $z_{1}^{\eta}, \ldots, z_{n}^{\eta}$ be space-time points in the rescaled gasket $\mathbb{L}_{\eta}=G_{m} \times 5^{-m} \mathbb{Z}$ such that $z_{1}^{\eta} \rightarrow z_{1}, \ldots, z_{n}^{\eta} \rightarrow z_{n}$ as $\eta \rightarrow 0$. Consider $n$ independent rescaled coalescing simple random walks in $G_{m}$ started from $z_{1}^{\eta}, \ldots, z_{n}^{\eta}$ and making jumps at times in $5^{-m} \mathbb{Z}$. Let $\left(Y_{1}^{\eta}, \ldots, Y_{n}^{\eta}\right)$, viewed as a random element of $\left(\Pi^{n}, \varrho^{n}\right)$ as defined in (2.5) with $d=2$, be the collection of $n$ continuous paths obtained by linearly interpolating the above $n$ coalescing random walks. Let $\left(Y_{1}, \ldots, Y_{n}\right)$ be a system of $n$ independent coalescing Brownian motions started from $z_{1}, \ldots, z_{n}$, also viewed as a random variable in $\left(\Pi^{n}, \varrho^{n}\right)$.

Proposition 6.6. As $\eta \rightarrow 0$,

$$
\left(Y_{1}^{\eta}, \ldots, Y_{n}^{\eta}\right) \rightarrow\left(Y_{1}, \ldots, Y_{n}\right)
$$

in distribution on $\left(\Pi^{n}, \varrho^{n}\right)$.

PROOF. We need the following two facts (6.5) and (6.6) in the proof. Let $\left(W_{k 5^{-m}}^{m}\right)_{k \geq 0}$ and $\left(Z_{k 5^{-m}}^{m}\right)_{k \geq 0}$ be two independent simple random walk on $G_{m}$ starting at time 0 and let

$$
\tau^{m}=\min \left\{k 5^{-m} \geq 0: W_{k 5^{-m}}^{m}=Z_{k 5^{-m}}^{m}\right\}
$$

denote their coalescence time. Then for all $\alpha>0$,

$$
\limsup _{m \rightarrow \infty} \sup _{x, y \in G_{m}:|x-y| \leq \varepsilon} \mathbb{P}\left(\tau^{m}>\alpha \mid W_{0}^{m}=x, Z_{0}^{m}=y\right) \rightarrow 0 \quad \text { as } \varepsilon \rightarrow 0 .
$$

Similarly, if $\left(W_{t}\right)_{t \geq 0}$ and $\left(Z_{t}\right)_{t \geq 0}$ are two independent Brownian motions on $G$ with coalescence time $\tau$, then for all $\alpha>0$,

$$
\sup _{x, y \in G:|x-y| \leq \varepsilon} \mathbb{P}\left(\tau>\alpha \mid W_{0}=x, Z_{0}=y\right) \rightarrow 0 \quad \text { as } \varepsilon \rightarrow 0 .
$$

Let us now prove (6.5). By scaling it is enough to show that

$$
\limsup _{m} \sup _{x, y \in G_{0}:|x-y| \leq 2^{m}} \mathbb{P}\left(\tau^{0}>5^{m+M} \mid W_{0}^{0}=x, Z_{0}^{0}=y\right) \rightarrow 0
$$

as $M \rightarrow \infty$. 
By Lemma 6.3, if $|x-y| \leq 2^{m}$, then

$$
\mathbb{P}\left(\tau^{0} \leq 5^{m} \mid W_{0}^{0}=x, Z_{0}^{0}=y\right) \geq c
$$

for some absolute positive constant $c$. Using the heat kernel upper bound (6.3), it is straightforward to show that given $\kappa>0$, there exists $K \in \mathbb{Z}_{+}$such that for all $m \geq 1$,

$$
\mathbb{P}\left(\left|W_{5^{m}}^{0}-W_{0}^{0}\right|>2^{m+K-2}\right) \leq \kappa .
$$

Fix $\varepsilon>0$ and let $L$ be such that $(1-c)^{L} \leq \varepsilon / 2$. Note that (6.8) and (6.9) with $\kappa=\varepsilon(4 L)^{-1}$ imply that when $m$ large enough, starting from $x, y \in G_{0}$ with $\mid x-$ $y \mid \leq 2^{m}$, with probability at least $c$, the random walks $W^{0}$ and $Z^{0}$ either hit each other by time $5^{m}$ or else they will be at most $2^{m}+2 \cdot 2^{m+K-2} \leq 2^{m+K}$ distance apart at time $5^{m}$, with probability at least $1-\varepsilon(2 L)^{-1}$. In the latter case, using the Markov property, again with probability at least $c$, the random walks $W^{0}$ and $Z^{0}$ either hit each other by the next $5^{m+K}$ amount of time, or else, they will be at most $2^{m+K}+2 \cdot 2^{m+2 K-2} \leq 2^{m+2 K}$ distance apart at time $5^{m}+5^{m+K}$ with probability at least $1-\varepsilon(2 L)^{-1}$. Repeat this procedure $L$ times to deduce that for $M=L K$, the probability in (6.7) is bounded above by $\varepsilon / 2+(1-c)^{L} \leq \varepsilon$ and hence (6.5) is proved.

To conclude (6.6) from (6.5), use the weak convergence random walk in the gasket toward Brownian motion, keeping in mind that the event $\{(f, g): f, g \in$ $C\left(\mathbb{R}_{+}\right), f(s)=g(s)$ for some $\left.s \in[0, \alpha]\right\}$ is closed in $C\left(\mathbb{R}_{+}\right) \times C\left(\mathbb{R}_{+}\right)$.

Now we are ready to prove Proposition 6.6. Recall $\eta=2^{-m}$. Let $X_{1}^{\eta}, \ldots, X_{n}^{\eta}$ be the continuous paths of the independent random walks on $G_{m}$ starting from $z_{1}^{\eta}, \ldots, z_{n}^{\eta}$. Similarly, $X_{1}, \ldots, X_{n}$ are independent Brownian motions on $G$ from $z_{1}, \ldots, z_{n}$. To keep things simple, we will assume $n=2$, but the argument can easily be extended to general $n>2$ by induction. By the invariance principle, $X_{i}^{\eta} \rightarrow X_{i}$ in distribution on $(\Pi, \varrho)$. By the Skorokhod representation theorem, we may assume that $\left(X_{1}^{\eta}, X_{2}^{\eta}\right) \rightarrow\left(X_{1}, X_{2}\right)$ almost surely on $\left(\Pi^{2}, \varrho^{2}\right)$. Let us show that $\varrho\left(Y_{2}^{\eta}, Y_{2}\right) \rightarrow 0$ in probability. It is enough to show for any $R>0$, $\left|\hat{Y}_{2}^{\eta}(t)-\hat{Y}_{2}(t)\right|_{L^{\infty}([-R, R])} \rightarrow 0$, where for $\left(\gamma, t_{0}\right) \in \Pi$, we denote by $\hat{\gamma}$ the continuous function that extends $\gamma$ to all of $\mathbb{R}$ by setting $\hat{\gamma}(t)=\gamma\left(t_{0}\right)$ for all $t<t_{0}$. Define

$$
\varepsilon_{\eta}=\left|\hat{X}_{1}^{\eta}(t)-\hat{X}_{1}(t)\right|_{L^{\infty}([-R, R])} \vee\left|\hat{X}_{2}^{\eta}(t)-\hat{X}_{2}(t)\right|_{L^{\infty}([-R, R])} .
$$

We have $\varepsilon_{\eta} \rightarrow 0$ almost surely. Let $\tau^{\eta}=\inf \left\{t \in 5^{-m} \mathbb{Z}: X_{1}^{\eta}(t)=X_{2}^{\eta}(t)\right\}$ and $\tau=$ $\inf \left\{t \in \mathbb{R}: X_{1}(t)=X_{2}(t)\right\}$. Now, we can estimate

$$
\begin{aligned}
\left|\hat{Y}_{2}^{\eta}(t)-\hat{Y}_{2}(t)\right|_{L^{\infty}([-R, R])} \leq & \varepsilon_{\eta}+\mathbf{1}_{\left\{\tau_{\eta}>\tau\right\}} \sup _{s \in\left[\tau, \tau_{\eta}\right] \cap[-R, R]}\left|X_{2}^{\eta}(s)-X_{1}(s)\right| \\
& +\mathbf{1}_{\left\{\tau_{\eta}<\tau\right\}} \sup _{s \in\left[\tau_{\eta}, \tau\right] \cap[-R, R]}\left|X_{1}^{\eta}(s)-X_{2}(s)\right|,
\end{aligned}
$$


which is bounded by

$$
\begin{aligned}
3 \varepsilon_{\eta}+ & \mathbf{1}_{\left\{\tau_{\eta}>\tau\right\}} \sup _{s \in\left[\tau, \tau_{\eta}\right] \cap[-R, R]}\left|X_{2}(s)-X_{1}(s)\right| \\
& +\mathbf{1}_{\left\{\tau_{\eta}<\tau\right\}} \sup _{s \in\left[\tau_{\eta}, \tau\right] \cap[-R, R]}\left|X_{1}(s)-X_{2}(s)\right| .
\end{aligned}
$$

Note that whenever one of the pairs $\left(X_{1}^{\eta}, X_{2}^{\eta}\right)$ or $\left(X_{1}, X_{2}\right)$ coalesces at some time within $[-R, R]$, at that moment the two processes of the other pair can be at distance at most $2 \varepsilon_{\eta}$ apart from each other. Hence, by (6.5) and (6.6), for any $\delta>0$, except for an event with probability at most $o_{\eta}(1),(6.10)$ can be bounded by

$$
3 \varepsilon_{\eta}+\sup _{s \in[\tau-\delta, \tau+\delta] \cap[-R, R]}\left|X_{1}(s)-X_{2}(s)\right| \leq 3 \varepsilon_{\eta}+\operatorname{osc}\left(X_{1}-X_{2} ; \delta\right),
$$

where for a function $\varphi$, its oscillation is defined as $\operatorname{osc}(\varphi ; \delta)=\sup \{\mid \varphi(s)-$ $\varphi(t)|: s, t \in[-R, R]| s-t \mid, \leq \delta\}$, and we have used the simple observation that $X_{1}(s)-X_{2}(s)=\left(X_{1}(s)-X_{2}(s)\right)-\left(X_{1}(\tau)-X_{2}(\tau)\right)$. Since, $X_{1}$ and $X_{2}$ are uniformly continuous on $[-R, R]$, almost surely $\operatorname{osc}\left(X_{1}-X_{2} ; \delta\right) \rightarrow 0$ as $\delta \rightarrow 0$. This proves Proposition 6.6.

This completes the proof of Theorem 6.1.

\section{APPENDIX: CONNECTION TO THE BROWNIAN WEB OF FONTES}

ET AL. [12]

We now briefly recall from [12] the construction of the Brownian web in the space of compact sets of continuous paths. Let $\mathbb{R}_{c}^{2}$ denote the completion of the space-time plane $\mathbb{R}^{2}$ with respect to the metric

$$
d\left(\left(x_{1}, t_{1}\right),\left(x_{2}, t_{2}\right)\right)=\left|\tanh \left(t_{1}\right)-\tanh \left(t_{2}\right)\right| \vee\left|\frac{\tanh \left(x_{1}\right)}{1+\left|t_{1}\right|}-\frac{\tanh \left(x_{2}\right)}{1+\left|t_{2}\right|}\right| .
$$

It is helpful to think of $\mathbb{R}_{c}^{2}$ as the continuous image of $[-\infty, \infty]^{2}$ under a map that identifies the lines $[-\infty, \infty] \times\{\infty\}$ and $[-\infty, \infty] \times\{-\infty\}$ with points $(*, \infty)$, and $(*,-\infty)$, respectively. Let $\hat{\Pi}$ be the space of all continuous paths in $\mathbb{R}_{c}^{2}$ with all possible starting times in $[-\infty, \infty]$. A continuous path $\gamma$ in $\mathbb{R}_{c}^{2}$ with starting point $\sigma_{\gamma} \in[-\infty, \infty]$, is a mapping $\gamma:\left[\sigma_{\gamma}, \infty\right] \rightarrow[-\infty, \infty] \cup\{*\}$ such that $\gamma(\infty)=*$, $\gamma\left(\sigma_{\gamma}\right)=*$ if $\sigma_{\gamma}=\infty$, and $t \mapsto(\gamma(t), t)$ is continuous from $\left[\sigma_{\gamma}, \infty\right] \rightarrow \mathbb{R}_{c}^{2}$. The space $\hat{\Pi}$ is equipped with the metric

$$
\begin{aligned}
\hat{\varrho}\left(\left(\gamma_{1}, \sigma_{\gamma_{1}}\right),\left(\gamma_{2}, \sigma_{\gamma_{2}}\right)\right)= & \left|\tanh \left(\sigma_{\gamma_{1}}\right)-\tanh \left(\sigma_{\gamma_{2}}\right)\right| \\
& +\sup _{t \geq \sigma_{\gamma_{1}} \wedge \sigma_{\gamma_{2}}}\left|\frac{\tanh \gamma_{1}\left(\left(t \vee \sigma_{\gamma_{1}}\right)\right)}{1+|t|}-\frac{\tanh \left(\gamma_{2}\left(t \vee \sigma_{\gamma_{2}}\right)\right)}{1+|t|}\right|,
\end{aligned}
$$


which makes $\hat{\Pi}$ a complete separable metric space. Now define $\mathcal{K}$ to be the space of compact subsets of $\hat{\Pi}$ and endow $\mathcal{K}$ with the standard Hausdorff metric $d_{\mathcal{K}}$. The space $\left(\mathcal{K}, d_{\mathcal{K}}\right)$ again turns out to be a complete separable metric space and the Brownian web can be defined as a random element in $\mathcal{K}$ via the following recipe.

Fix a countable ordered set $\mathcal{D}=\left(z_{1}, z_{2}, \ldots\right)$, which is dense in $\mathbb{R}^{2}$. Let $\mathcal{B}_{n}:=$ $\left\{W_{1}^{c}, \ldots, W_{n}^{c}\right\}$ be the set of $n$ coalescing Brownian paths starting from the spacetime points $z_{1}, \ldots, z_{n}$ as in Section 3.1, viewed as a random element in $\mathcal{K}$. In [12], the authors showed that almost surely, $\mathcal{B}_{n}$ converges in $\mathcal{K}$ to some random element $\mathcal{B}_{\infty}$, which they called the Brownian web. Its distribution does not depend on the choice of $\mathcal{D}$. Let $\mathcal{W}_{\infty}$ be distributed according to the coalescing Brownian flow on $\mathscr{H}$. It is natural to ask how these two different objects $\mathcal{B}_{\infty}$ and $\mathcal{W}_{\infty}$ are related.

For $\left(\gamma, \sigma_{\gamma}\right) \in \hat{\Pi}$, the notion of a tube $T$ being crossed (or traversed) by $\left(\gamma, \sigma_{\gamma}\right)$ remains exactly the same. Namely, a tube $T$ is crossed by $\left(\gamma, \sigma_{\gamma}\right)$ if $\sigma_{\gamma} \leq t_{0}$, $\left(\gamma\left(t_{0}\right), t_{0}\right) \in \partial_{0} T,\left(\gamma\left(t_{1}\right), t_{1}\right) \in \partial_{1} T$ and $(\gamma(s), s) \in[T]$ for all $s \in\left(t_{0}, t_{1}\right)$, where $t_{0}$ and $t_{1}$ be the start time and the end time of $T$, respectively. For a subset $F$ of $\hat{\Pi}$, let $\hat{\operatorname{Cr}}(F)$ denote the set of tubes in $\mathcal{T}$ which are crossed by at least one path in $F$. Clearly, $\hat{\operatorname{Cr}}(F)$ is always hereditary. The exact same proof of Lemma 2.9 shows that $\hat{\operatorname{Cr}}(F)$ is closed in $\mathcal{T}$ if $F$ is compact. This means $\hat{C}$ r maps $\mathcal{K}$ into $\mathscr{H}$.

THEOREM A.1. We have

$$
\hat{\operatorname{Cr}}\left(\mathcal{B}_{\infty}\right) \stackrel{d}{=} \mathcal{W}_{\infty}
$$

The above theorem says that the law of the coalescing Brownian flow on $\mathbb{R}$ in the tube topology is nothing but the push-forward of the law of the Brownian web in the path space. It gives another construction of the coalescing Brownian flow on $\mathbb{R}$, though the similar construction does not work for the gasket due to the absence of appropriate 'Brownian web on the gasket'.

Proof of TheOREM A.1. Let $\mathcal{W}_{n}=\mathcal{W}\left(z_{1}, \ldots, z_{n}\right) \in \mathscr{H}$ be as defined in Section 3.1. Clearly, $\hat{\operatorname{Cr}}\left(\mathcal{B}_{n}\right)=\operatorname{Cr}\left(\mathcal{B}_{n}\right)=\mathcal{W}_{n}$. We have seen in the proof of Theorem 3.1 that

$$
\mathcal{W}_{n} \stackrel{d}{\rightarrow} \mathcal{W}_{\infty} \quad \text { in }\left(\mathscr{H}, d_{\mathscr{H}}\right)
$$

Hence, the proof of the theorem is complete by Lemma A.2 and the continuous mapping theorem.

Lemma A.2. The map $\hat{\mathrm{Cr}}:\left(\mathcal{K}, d_{\mathcal{K}}\right) \rightarrow\left(\mathscr{H}, d_{\mathscr{H}}\right)$ is continuous.

ProOF. Let $F_{n} \rightarrow F$ in Hausdorff metric $d_{\mathcal{K}}$. A basis element in the topol-

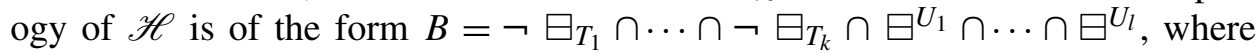
$T_{1}, \ldots, T_{k} \in \mathcal{T}$ and $U_{1}, \ldots, U_{l}$ are open sets in $\mathcal{T}$. We need to show that if $H:=\hat{\operatorname{Cr}}(F) \in B$, then $H_{n}:=\hat{\operatorname{C}}\left(F_{n}\right) \in B$ for sufficiently large $n$. 
Note that $H \in \boxminus^{U_{i}}$ means that there is a $T \in U_{i}$ such that $T$ is traversed by some $(f, t) \in F$. Since $U_{i}$ is open, we can find another tube $T^{\prime} \in U_{i}$ such that $T^{\prime}<T$. Moreover, there exists $\left(f_{n}, t_{n}\right) \in F_{n}$ such that $\varrho\left(\left(f_{n}, t_{n}\right),(f, t)\right) \leq 2 d_{\mathcal{K}}\left(F_{n}, F\right) \rightarrow$ 0 . Clearly, $T^{\prime}$ is traversed by $\left(f_{n}, t_{n}\right)$ for all $n \geq n_{0}$, and hence, $H_{n} \in \boxminus^{U_{i}}$ for all $n \geq n_{0}$.

Next, we have to argue that if $T \in \mathcal{T}$ is not traversed by $F$, then $T \in \mathcal{T}$ is not traversed by $F_{n}$ for sufficiently large $n$. Suppose that $T$ is traversed by $\left(f_{n}, t_{n}\right) \in F_{n}$ infinitely often, then it suffices to prove that $T$ is traversed by $F$ as well. For notational convenience, we will assume that $T$ is traversed by $\left(f_{n}, t_{n}\right) \in F_{n}$ for all $n$. Using compactness of $F$ and the fact that $d_{\mathcal{K}}\left(F_{n}, F\right) \rightarrow 0$, we can find $(f, t) \in F$ such that $\varrho\left(\left(f_{n}, t_{n}\right),(f, t)\right) \rightarrow 0$. It is now easy to check, along the lines of the proof of Lemma 2.9, that $T$ is traversed by $(f, t) \in F$. This completes the proof.

REMARK A.3. By Lemma A.2, the pre-image of any event in the tube topology under the map $\hat{C} r$ is measurable in the path topology. So, the tube topology is weaker (or coarser) than the path topology. It would be interesting to find examples of events which are measurable in the path topology but whose images under $\hat{C} r$ are not measurable in the tube topology.

\section{REFERENCES}

[1] Aizenman, M. and Burchard, A. (1999). Hölder regularity and dimension bounds for random curves. Duke Math. J. 99 419-453. MR1712629

[2] Angel, O., Berestycki, N. and Limic, V. (2012). Global divergence of spatial coalescents. Probab. Theory Related Fields 152 625-679. MR2892958

[3] Arratia, R. (1981). Coalescing Brownian motions and the voter model on $\mathbb{Z}$. Unpublished partial manuscript. Available from rarratia@math.usc.edu.

[4] Arratia, R. A. (1979). Coalescing Brownian motions on the line. Ph.D. Thesis, Univ. Wisconsin, Madison. MR2630231

[5] BARLOW, M. T. (1998). Diffusions on fractals. In Lectures on Probability Theory and Statistics (Saint-Flour, 1995). Lecture Notes in Math. 1690 1-121. Springer, Berlin. MR1668115

[6] BArlow, M. T. and Perkins, E. A. (1988). Brownian motion on the Sierpiński gasket. Probab. Theory Related Fields 79 543-623. MR0966175

[7] Belhaouari, S., Mountford, T., Sun, R. and Valle, G. (2006). Convergence results and sharp estimates for the voter model interfaces. Electron. J. Probab. 11 768-801 (electronic). MR2242663

[8] Berestycki, N., Garban, C. and Sen, A. (2015). A new approach to coalescing Brownian flows II: Black noise property. In preparation.

[9] Camia, F. and Newman, C. M. (2006). Two-dimensional critical percolation: The full scaling limit. Comm. Math. Phys. 268 1-38. MR2249794

[10] Coletti, C. F., Fontes, L. R. G. and Dias, E. S. (2009). Scaling limit for a drainage network model. J. Appl. Probab. 46 1184-1197. MR2582714

[11] Evans, S. N., Morris, B. and SEN, A. (2013). Coalescing systems of non-Brownian particles. Probab. Theory Related Fields 156 307-342. MR3055261

[12] Fontes, L. R. G., Isopi, M., Newman, C. M. and Ravishankar, K. (2004). The Brownian web: Characterization and convergence. Ann. Probab. 32 2857-2883. MR2094432 
[13] Garban, C., Pete, G. and Schramm, O. (2013). Pivotal, cluster, and interface measures for critical planar percolation. J. Amer. Math. Soc. 26 939-1024. MR3073882

[14] Garban, C., Pete, G. and Schramm, O. (2013). The scaling limits of near-critical and dynamical percolation. Preprint. Available at arXiv:1305.5526.

[15] Jones, O. D. (1996). Transition probabilities for the simple random walk on the Sierpiński graph. Stochastic Process. Appl. 61 45-69. MR1378848

[16] LE JAN, Y. (2006). New developments in stochastic dynamics. In International Congress of Mathematicians. Vol. III 649-667. Eur. Math. Soc., Zürich. MR2275701

[17] Le JAN, Y. and Lemaire, S. (2004). Products of Beta matrices and sticky flows. Probab. Theory Related Fields 130 109-134. MR2092875

[18] Le Jan, Y. and RAimond, O. (2004). Flows, coalescence and noise. Ann. Probab. 32 12471315. MR2060298

[19] Le Jan, Y. and Raimond, O. (2004). Sticky flows on the circle and their noises. Probab. Theory Related Fields 129 63-82. MR2052863

[20] Newman, C. M., Ravishankar, K. and Sun, R. (2005). Convergence of coalescing nonsimple random walks to the Brownian web. Electron. J. Probab. 10 21-60. MR2120239

[21] NorRIS, J. and TURNER, A. (2012). Hastings-Levitov aggregation in the small-particle limit. Comm. Math. Phys. 316 809-841. MR2993934

[22] SARKAR, A. and SUN, R. (2013). Brownian web in the scaling limit of supercritical oriented percolation in dimension 1+1. Electron. J. Probab. 18 no. 21, 23. MR3035749

[23] Schramm, O. and Smirnov, S. (2011). On the scaling limits of planar percolation. Ann. Probab. 39 1768-1814. MR2884873

[24] SPitZer, F. (1976). Principles of Random Walk, 2nd ed. Springer, New York. MR0388547

[25] Tóth, B. and Werner, W. (1998). The true self-repelling motion. Probab. Theory Related Fields 111 375-452. MR1640799

N. BERESTYCKI

STATISTICAL LABORATORY

DPMMS

UNIVERSITY OF CAMBRIDGE

WILBERFORCE RD.

CAMBRIDGE, CB3 0WB

UNITED KINGDOM

E-MAIL: nberestycki@gmail.com
C. GARBAN

INSTITUT CAMILLE JORDAN

UNIVERSITÉ LYON 1

43 BD DU 11 NOVEMBRE 1918

69622 VILLEURBANNE CEDEX

FRANCE

E-MAIL: garban@math.univ-lyon1.fr

\author{
A. SEN \\ DEPARTMENT OF MATHEMATICS \\ UNIVERSITY OF MINNESOTA \\ 127 VINCENT HALL \\ 206 Church ST. SE \\ MinNEAPOLIS, MinNESOTA 55455 \\ USA \\ E-MAIL: arnab@umn.edu
}

